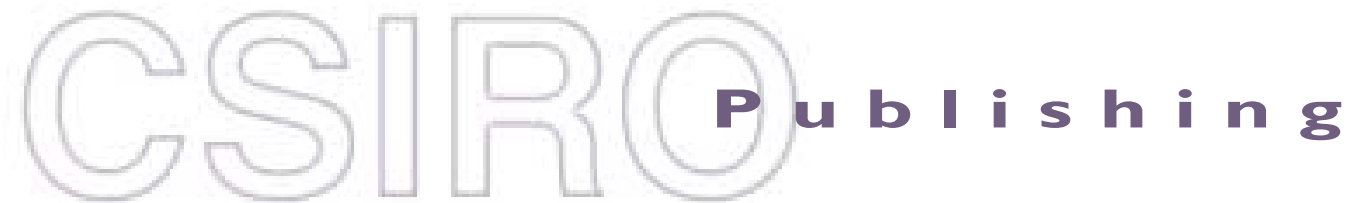

\section{Publications of the Astronomical Society of Australia}

Volume 19, 2002

(C) Astronomical Society of Australia 2002

An international journal of astronomy and astrophysics

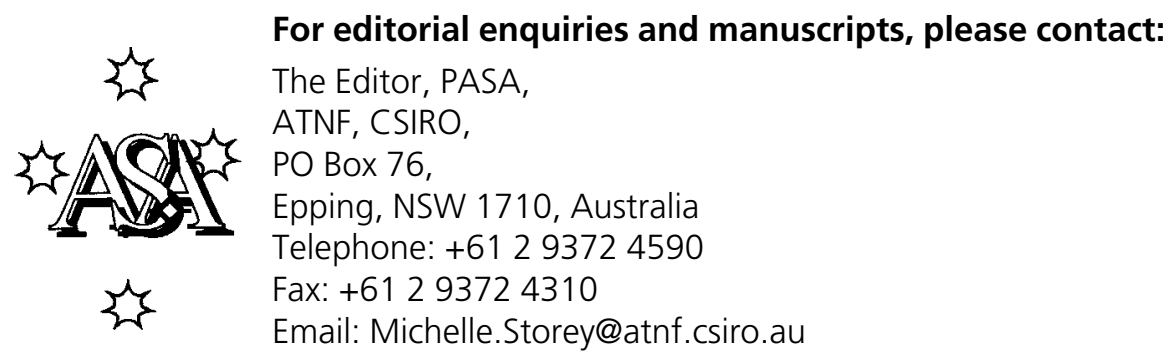

For general enquiries and subscriptions, please contact: CSIRO Publishing PO Box 1139 (150 Oxford St)

Collingwood, Vic. 3066, Australia

Telephone: +6139662 7666

Fax: +61 396627555

Email: publishing.pasa@csiro.au

C S I RO

PUBLISHING Published by CSIRO Publishing

for the Astronomical Society of Australia

www.publish.csiro.au/journals/pasa 


\title{
Evolution of the Radio Remnant of SN 1987A: 1990-2001
}

\author{
R. N. Manchester ${ }^{1}$, B. M. Gaensler ${ }^{2}$,*, V. C. Wheaton ${ }^{1,3}$, L. Staveley-Smith ${ }^{1}$, \\ A. K. Tzioumis ${ }^{1}$, N. S. Bizunok ${ }^{2,4}$, M. J. Kesteven ${ }^{1}$ and J. E. Reynolds ${ }^{1}$ \\ ${ }^{1}$ Australia Telescope National Facility, CSIRO, PO Box 76, Epping NSW 1710 \\ Dick.Manchester@csiro.au \\ ${ }^{2}$ Center for Space Research, Massachusetts Institute of Technology, Cambridge MA 02139, USA \\ ${ }^{3}$ School of Physics, University of Sydney, NSW 2006 \\ ${ }^{4}$ Boston University, Boston MA 02215, USA \\ Received 2001 July 13, accepted 2001 October 31
}

\begin{abstract}
The development of the radio remnant of SN 1987A has been followed using the Australia Telescope Compact Array since its first detection in 1990 August. The remnant has been observed at four frequencies, $1.4,2.4,4.8$, and $8.6 \mathrm{GHz}$, at intervals of $4-6$ weeks since the first detection. These data are combined with the $843 \mathrm{MHz}$ data set of Ball et al. (2001) obtained at Molonglo Observatory to study the spectral and temporal variations of the emission. These observations show that the remnant continues to increase in brightness, with a larger rate of increase at recent times. They also show that the radio spectrum is becoming flatter, with the spectral index changing from -0.97 to -0.88 over the 11 years. In addition, at roughly yearly intervals since 1992, the remnant has been imaged at $9 \mathrm{GHz}$ using super-resolution techniques to obtain an effective synthesised beamwidth of about 0.5 . The imaging observations confirm the shell morphology of the radio remnant and show that it continues to expand at $\sim 3000 \mathrm{~km} \mathrm{~s}^{-1}$. The bright regions of radio emission seen on the limb of the shell do not appear to be related to the optical hot spots which have subsequently appeared in surrounding circumstellar material.
\end{abstract}

Keywords: circumstellar matter — radio continuum: ISM — supernovae: individual (SN 1987A) — supernova remnants

\section{Introduction}

As the closest observed supernova in nearly 400 years, SN 1987A in the Large Magellanic Cloud offers unique opportunities for detailed study of the evolution of a supernova and the birth of a supernova remnant. In the 14 years since the explosion was detected, it has been extensively studied in all wavelength bands from radio to gamma ray using both ground- and space-based observatories. These observations have revealed a complex evolution of both the supernova itself and the supernova remnant which is developing as the ejecta and their associated shocks interact with the circumstellar material.

Perhaps the most dramatic of these observations are the optical observations which show the beautiful tripleringed structure surrounding and illuminated by the supernova (Burrows et al. 1995). The inner ring is believed to represent an equatorial density enhancement in the circumstellar gas at the interface between a dense wind emitted from an earlier red giant phase of the supernova progenitor star, $\mathrm{Sk}-69^{\circ} 202$, and a faster wind emitted by this star in more recent times (Crotts, Kunkel, \& Heathcote 1995; Plait et al. 1995). In the past few years, there has been increasing evidence for interaction of the expanding ejecta or the associated shocks with the equatorial ring, with small regions of enhanced $\mathrm{H} \alpha$ emission, or 'hot spots', appearing just inside the ring. The first of these was detected by Pun et al. (1997), but studies of ground-based

\footnotetext{
${ }^{*}$ Hubble Fellow. Present address: Harvard-Smithsonian Centre for Astrophysics, Cambridge MA 02138, USA.
}

and Hubble Space Telescope (HST) data by Lawrence et al. (2000) show that this spot was detectable as far back as March 1995 (day 2933 since the supernova ${ }^{1}$ ). Lawrence et al. (2000) also present evidence for up to eight additional regions of enhanced emission from about day 4300 (December 1998). The most prominent are the original spot at position angle $29^{\circ}$ and a group of spots just south of east between position angles of $90^{\circ}$ and $140^{\circ}$.

At radio wavelengths, the initial outburst was very short-lived (Turtle et al. 1987) compared to other radio supernovae (e.g. Weiler et al. 1998). This prompt outburst is attributed to shock acceleration of synchrotron-emitting electrons in the stellar wind close to the star at the time of the explosion (Storey \& Manchester 1987; Chevalier \& Fransson 1987). After about 3 years, in mid-1990, radio emission was again detected from the supernova, with the Molonglo Observatory Synthesis Telescope (MOST) at $843 \mathrm{MHz}$ (Ball et al. 1995) and with the Australia Telescope Compact Array (ATCA) at 1.4, 2.4, 4.8, and $8.6 \mathrm{GHz}$ (Staveley-Smith et al. 1992; Gaensler et al. 1997). This emission has increased more or less monotonically since its first detection. The spectral index over the observed frequency range has remained close to -0.9 , indicating optically thin synchrotron emission. X-ray emission was observed to turn on at about the same time as the second phase of radio emission and also has increased in intensity since then (Gorenstein, Hughes, \& Tucker 1994; Hasinger, Aschenbach, \& Trümper 1996). Recent observations with

\footnotetext{
${ }^{1}$ Day number $=$ MJD -46849.3.
} 
the Chandra X-ray Observatory have resolved the X-ray emission into an approximately circular shell (Burrows et al. 2000).

This second phase of increasing emission is attributed to the interaction of shocks driven by the ejecta with circumstellar material and is distinct from the interaction with a radially decreasing stellar wind which characterises radio supernovae. It therefore signifies the birth of the remnant of SN 1987A - the first observation of the birth of a supernova remnant. We use the name SNR 1987A for the remnant.

By late 1992, SNR 1987A was sufficiently strong to image at $9 \mathrm{GHz}$ using the ATCA (Staveley-Smith et al. 1993a). This image, which exploited super-resolution to resolve the source, showed that the remnant was roughly circular with a diameter of about 0 ". 8 , fitting inside the optical equatorial ring (Reynolds et al. 1995), and with bright lobes to the east and west, suggesting an annular structure with an axis similar to that of the optical emission. The eastern lobe was about $20 \%$ brighter than the western lobe. To follow the evolution of this structure, the remnant has been imaged at roughly yearly intervals since 1992.

Despite the increasing brightness, the size of SNR 1987A is increasing only slowly. Gaensler et al. (1997) fitted a model consisting of a thin spherical shell to the $u v$-plane data and showed that, between 1992 and 1995, the average expansion velocity of the remnant was only $2800 \pm 400 \mathrm{~km} \mathrm{~s}^{-1}$. In contrast, the average expansion speed between 1987 and 1991 was about $35,000 \mathrm{~km} \mathrm{~s}^{-1}$ (cf. Jauncey et al. 1988). Comparison of four images obtained between 1992 and 1995 showed that the brightness of the lobes increased relative to that of the spherical shell and that the asymmetry between the east and west lobes increased markedly over this period (Gaensler et al. 1997). By 1995, the peak brightness of the eastern lobe was 1.8 times that of the western lobe.

The only other young supernova which has been imaged with high resolution at radio wavelengths is $\mathrm{SN}$ 1993J in M81. Frequent observations using Very Long Baseline Interferometry (VLBI) techniques (e.g. van Dyk et al. 1994; Marcaide et al. 1997; Bartel et al. 2000) have shown that the radio emission is in the form of an expanding shell, the outline of which is nearly circular, but rather lumpy. Unlike SNR 1987A, SN 1993J remains in the radio supernova stage with decreasing flux density, making further imaging difficult. Recent observations of SN 1980K (Montes et al. 1998) and SN 1979C (Montes et al. 2000) have shown variations in the rate of decline of flux density or, in the case of SN 1979C, possibly small increases, indicating variations in the density of the circumstellar medium, but, like SN 1993J, these objects are best considered to be still in their radio supernova stage.

The slow expansion velocity of SNR 1987A suggests that the expanding shock and the leading ejecta have encountered a significant density enhancement, greatly reducing their velocity (Chevalier 1992; Duffy, Ball, \& Kirk 1995; Chevalier \& Dwarkadas 1995). These models assume spherical symmetry and hence do not account for the increasing asymmetry of the source. Furthermore, they do not predict the continuing increase in brightness of the remnant as observed by Ball et al. (1995) and discussed further below. An alternative explanation for the slow apparent expansion velocity is that the shock excites slowly moving clumps of circumstellar material and then moves on. Ball \& Kirk (1992) modelled the emission observed up to day 1800 by shock heating of two clumps and obtained a good fit to the data up to that time.

As discussed by Gaensler et al. (1997), it is not easy to account for the observed asymmetry of SNR 1987A. Models involving the annular structure of the circumstellar material (e.g. Chevalier \& Dwarkadas 1995) have difficulty accounting for the degree of enhancement in the lobes and the east-west asymmetry. Similar difficulties are encountered in trying to explain the optical hot spots. Lawrence et al. (2000) conclude that "fingers or jets" in the distribution of ejecta from the supernova is the most plausible explanation.

In a recent publication, Ball et al. (2001) have analysed the $843 \mathrm{MHz}$ flux densities observed with MOST to 2000 May (day 4820). They find a transition from a declining rate of increase observed from about mid1991 (day 1600) to early 1995 (day 2900), to a larger and constant rate of increase, $62.7 \pm 0.5 \mu \mathrm{Jy}$ day $^{-1}$, since then. This change in slope occurred at about the same time as the appearance of the first optical hot spot (Lawrence et al. 2000), suggesting a possible connection.

In this paper, we extend the ATCA observational database from 1995 to February 2001 (day 5100) and discuss these results in conjunction with those of Ball et al. (2001). The evolution of the radio flux densities is discussed in Section 2. The sequence of images is extended to late 2000 and discussed in relation to recent optical and X-ray imaging in Section 3. Future prospects are canvassed in Section 4.

\section{Evolution of Radio Flux Densities and Spectral Index}

Flux density monitoring observations of SNR 1987A are made using the ATCA at 4-6 week intervals using one of the $6 \mathrm{~km}$ array configurations. Observations are made simultaneously at two frequencies, either 1380 and $2496 \mathrm{MHz}$ (2368 MHz before mid-1997) or 4790 and $8640 \mathrm{MHz}$. A $128 \mathrm{MHz}$ bandwidth is observed at all frequencies. The two frequency pairs are observed alternately, with $20 \mathrm{~min}$ on SNR 1987A and $3 \mathrm{~min}$ on phase calibrators before and after the SNR observation, with a total observation time typically of $12 \mathrm{~h}$. All observations are made with a $\mathbf{J} 2000$ pointing and phase centre of RA $05^{\mathrm{h}} 35^{\mathrm{m}} 27^{\mathrm{S}} .90$, Dec $-69^{\circ} 16^{\prime} 21^{\prime \prime}$. 6 , approximately $10^{\prime \prime}$ south of the SN 1987A position (Reynolds et al. 1995). The phase calibrators are PKS B0530-727, PKS B0407-658, and (at 4790/8640 MHz) PKS B0454-810. Flux calibration is relative to PKS B1934-638, assumed to have flux densities of $14.95,11.14,5.83$, and $2.84 \mathrm{Jy}$ at the four frequencies, respectively. 

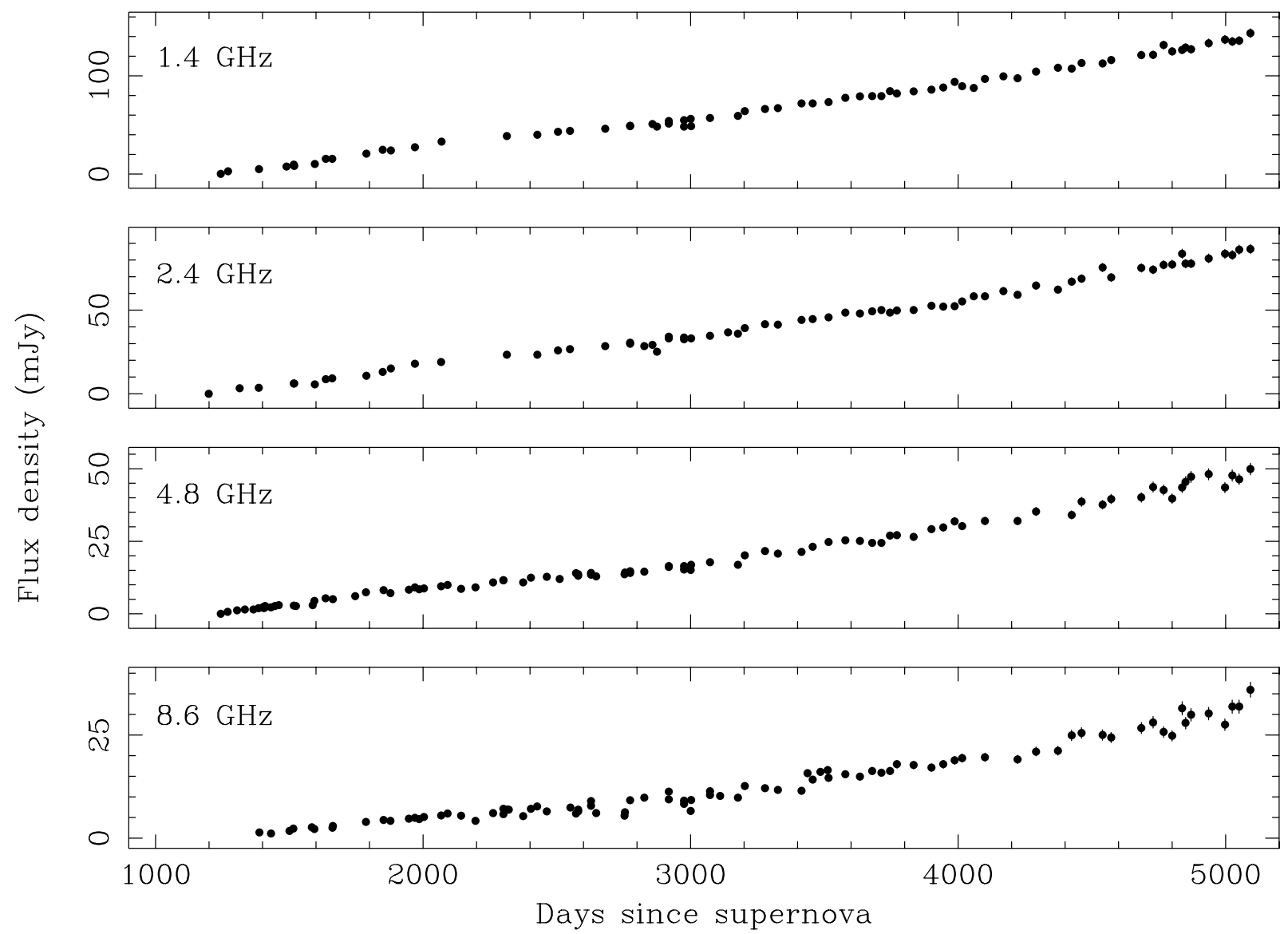

Figure 1 Flux densities of SNR 1987A from 1990 August to 2001 February observed with the Australia Telescope Compact Array.

Data are first checked for obvious interference or telescope problems, flagged if necessary and then are processed using MIRIAD ${ }^{2}$ scripts to ensure consistency. Visibility data are calibrated in both phase and amplitude and images formed using baselines longer than $3 \mathrm{k} \lambda$. These images are cleaned and sources above a threshold identified. A table of source positions and integrated and peak flux densities is output for each frequency. At least at the two higher frequencies, SNR 1987A is resolved and so integrated flux densities are quoted. An unresolved source, J0536-6919, is present on all images with an observed flux density ranging between $80 \mathrm{mJy}$ at $1.4 \mathrm{GHz}$ and $6 \mathrm{mJy}$ at $8.6 \mathrm{GHz}$ and serves as a check on the system calibration. Its $\mathrm{J} 2000$ position determined from the $4.8 \mathrm{GHz}$ observations since day 4000 is RA $05^{\mathrm{h}} 36^{\mathrm{m}} 04^{\mathrm{s}} .789 \pm 0.005$, Dec $-69^{\circ} 18^{\prime} 44^{\prime \prime} .81 \pm 0^{\prime} .02$. At $8.6 \mathrm{GHz}$ it lies at about 1.5 primary beam radii from the pointing centre, so its flux density measurements at this frequency are not very reliable. Measured flux densities for SNR 1987A are given in Appendix 1.

Figure 1 shows the measured flux densities of SNR 1987A at the four ATCA frequencies. Estimated uncertainties represent a combination of random noise and scale errors resulting from errors in the calibration. Except at $8.6 \mathrm{GHz}$, the scale errors are estimated from the scatter

\footnotetext{
${ }^{2}$ See http://www.atnf.csiro.au/computing/software/miriad/
}

in the measured flux densities of J0536-6918 since day 4000. At $8.6 \mathrm{GHz}$ scale errors are taken to be 1.25 times the $4790 \mathrm{MHz}$ scale errors. At the lower frequencies and at later times, the errors in the flux density estimations are dominated by the scale errors. Since, except at $8.6 \mathrm{GHz}$, the flux density of J0536-6918 is comparable to that of SNR 1987A and since there is no evidence for systematic changes in the flux density of J0536-6918, these scale errors can be reduced by dividing the flux densities by the normalised flux density of J0536-6918 from the same observation. Flux densities from day 3000 scaled in this way for 1.4, 2.4, and $4.8 \mathrm{GHz}$ are shown in Figure 2.

These plots show that the continued increase in flux density observed at $843 \mathrm{MHz}$ by Ball et al. (2001) is also observed at higher frequencies. The increase in slope observed at about day 2900 in the MOST data is also seen in the ATCA data (Figure 1). Ball et al. (2001) stated that the MOST data after day 3000 were well fitted by a linear trend. However, we believe that there is significant evidence for a long-term increase in slope after day 3000 in both the MOST data set and the ATCA data sets, i.e. the rate of increase in flux density is increasing. Evidence for this is shown in Figure 3 which shows residual flux densities after fitting a second-order polynomial to the MOST and scaled ATCA flux densities from day 3000 and subtracting the linear component. These plots all show a systematic trend with the residual flux densities being, 


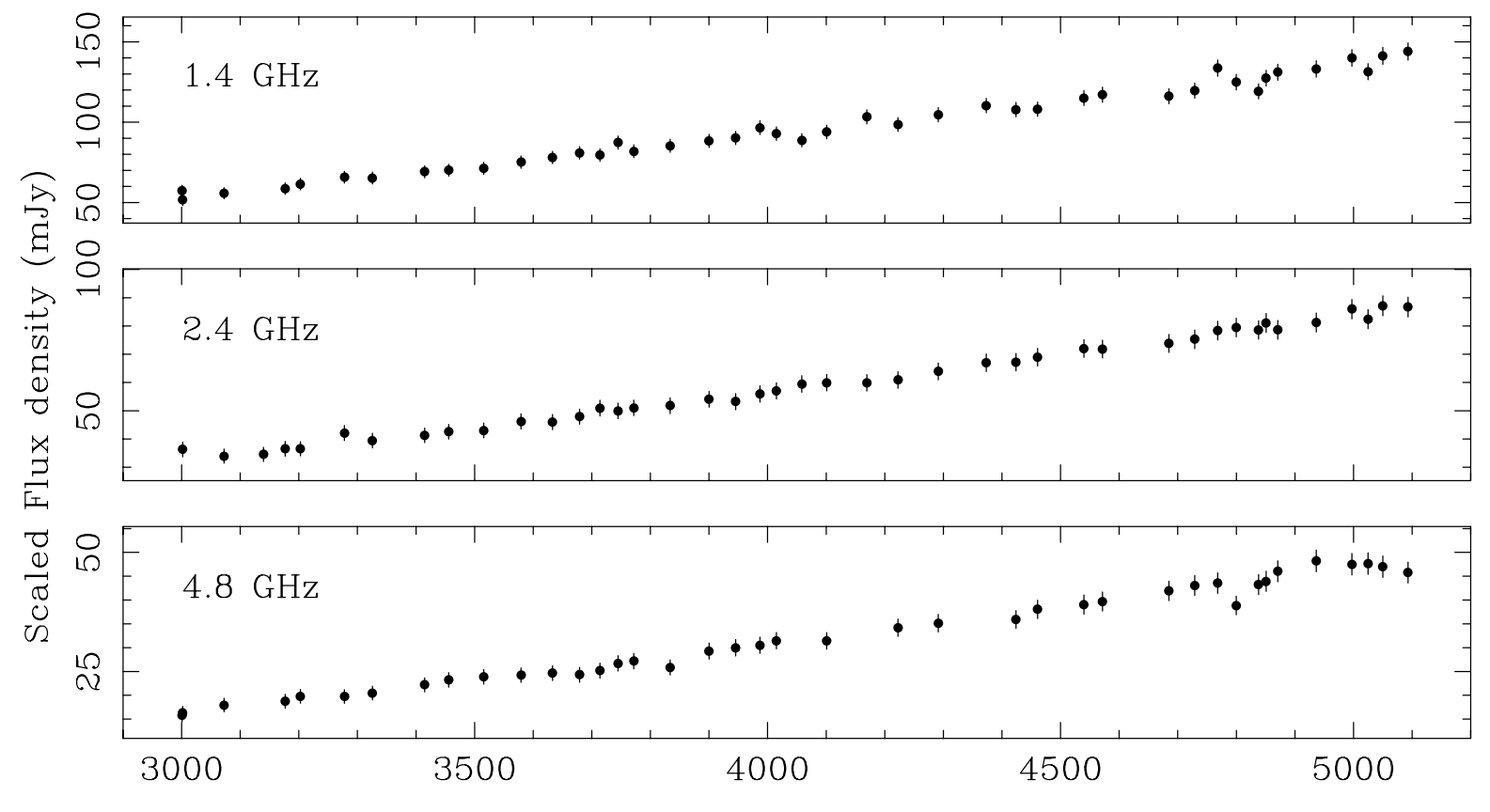

Days since supernova

Figure 2 Flux densities of SNR 1987A at 1.4, 2.4, and 4.8 GHz from 1995 May to 2001 February, scaled by the flux density of J0536-6918 obtained from the same image. This scaling removes most of the effects of mis-calibration.
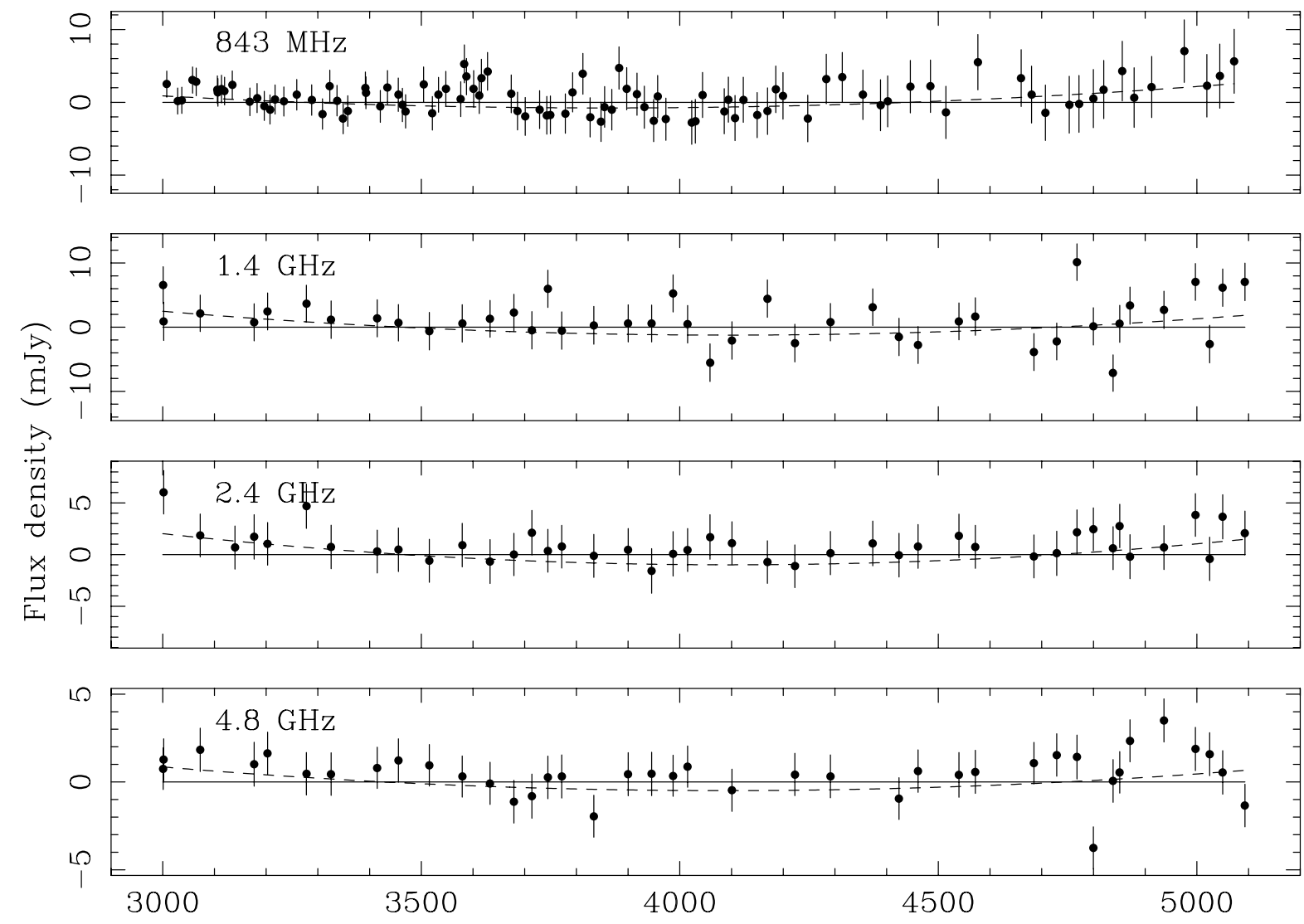

Days since supernova

Figure 3 Residual flux densities of SNR 1987A from 1990 August to 2001 February after fitting a second-order polynomial to the data and subtracting the constant and linear terms. The upper panel shows data from the MOST (Ball et al. 2001) and the lower three panels show ATCA data scaled by the J0536-6918 flux density. In each case the second-order term is shown by a dashed line. 
Table 1. Flux density gradients since day $\mathbf{3 0 0 0}$

\begin{tabular}{lcccccc}
\hline $\begin{array}{l}\text { Frequency } \\
(\mathrm{GHz})\end{array}$ & $\begin{array}{c}\text { Gradient } \\
(\mu \mathrm{Jy} / \text { day })\end{array}$ & $\begin{array}{c}\text { Intercept } \\
(\text { day })\end{array}$ & $\begin{array}{c}\text { Gradient }(<4050) \\
(\mu \mathrm{Jy} / \text { day })\end{array}$ & $\begin{array}{c}\text { Gradient }(>4050) \\
(\mu \mathrm{Jy} / \text { day })\end{array}$ & $\begin{array}{c}\text { Gradient change } \\
(\mu \mathrm{Jy} / \text { day })\end{array}$ & Reduced $\chi^{2}$ \\
\hline Unscaled & & & & & & \\
0.843 & $63.1 \pm 0.6$ & $1640 \pm 20$ & $61.4 \pm 1.0$ & $66.9 \pm 2.1$ & $5.5 \pm 2.3$ & 0.5 \\
1.4 & $40.9 \pm 0.8$ & $1750 \pm 50$ & $37.7 \pm 1.7$ & $46.6 \pm 2.4$ & $8.8 \pm 2.9$ & 0.7 \\
2.4 & $25.2 \pm 0.5$ & $1780 \pm 50$ & $20.7 \pm 1.2$ & $28.0 \pm 1.6$ & $7.3 \pm 1.9$ & 0.7 \\
4.8 & $15.2 \pm 0.4$ & $2010 \pm 55$ & $14.2 \pm 0.6$ & $17.5 \pm 1.2$ & $3.2 \pm 1.4$ & 1.3 \\
8.6 & $10.6 \pm 0.4$ & $2160 \pm 65$ & $10.3 \pm 0.8$ & $13.3 \pm 1.7$ & $3.0 \pm 1.9$ & 0.8 \\
Scaled by J0536-6918 & & & & & \\
1.4 & $41.2 \pm 0.7$ & $1770 \pm 45$ & $39.8 \pm 2.0$ & $46.3 \pm 2.0$ & $6.4 \pm 2.8$ & 1.3 \\
2.4 & $26.0 \pm 0.5$ & $1840 \pm 45$ & $22.8 \pm 1.5$ & $27.8 \pm 1.4$ & $5.0 \pm 2.0$ & 0.4 \\
4.8 & $15.3 \pm 0.3$ & $2020 \pm 45$ & $13.9 \pm 0.8$ & $15.5 \pm 1.0$ & $2.5 \pm 1.3$ & 1.0 \\
\hline
\end{tabular}

on average, negative at central times and positive at both early and late times. Figure 3 also shows the second-order term of the fit which in all cases is positive and of about $3 \sigma$ significance. These data sets are essentially independent so there can be little doubt about the significance of the effect.

To further quantify this effect, straight lines were fitted to the ATCA data sets from day 3000 , both for the unscaled data and for the data (except at $8.6 \mathrm{GHz}$ ) scaled by the flux density of J0536-6918. Results of this fitting are given in Table 1 . The second and third columns give the gradient and $x$-intercept of the lines fitted to all data between day 3000 and day 5100 (cf. Figure 3). The point of interest here is that there is a systematic increase of the date of intercept with frequency. Especially for the higher frequencies, the intercept is well after the date of first detection of the SNR. This shows that the higher frequencies have a higher relative rate of flux density increase and that the present rate of increase is much higher than that at early times. Note that this effect is seen in both the scaled and unscaled data, so it is not an artifact of the scaling procedure.

As a further check, the data sets were split into two halves, from day 3000 to day 4050 and from day 4050 to day 5100, and straight lines fitted to both halves. Results are tabulated in columns 4-7 of Table 1. The values of reduced $\chi^{2}$ for the combined fit were computed by treating the two lines as a single model describing the complete data set from day 3000 onward. These show that, independently at all frequencies, the change in gradient is significant at about the $2 \sigma$ level, and that this gradient increase is present in the MOST data and in both the scaled and the unscaled ATCA data.

Apparently significant short-term variations are seen, especially at $4.8 \mathrm{GHz}$, with a timescale of order 100 days. The clearest example is near the end of the $4.8 \mathrm{GHz}$ data set and is best seen in Figure 3. This fluctuation is not obvious at lower frequencies, but could be masked by the low signal-to-noise ratio. If real, these shortterm fluctuations imply significant interactions on a scale of $0.03 \mathrm{pc}\left(0^{\prime \prime} .1\right)$ or less, and furthermore, many such interactions.

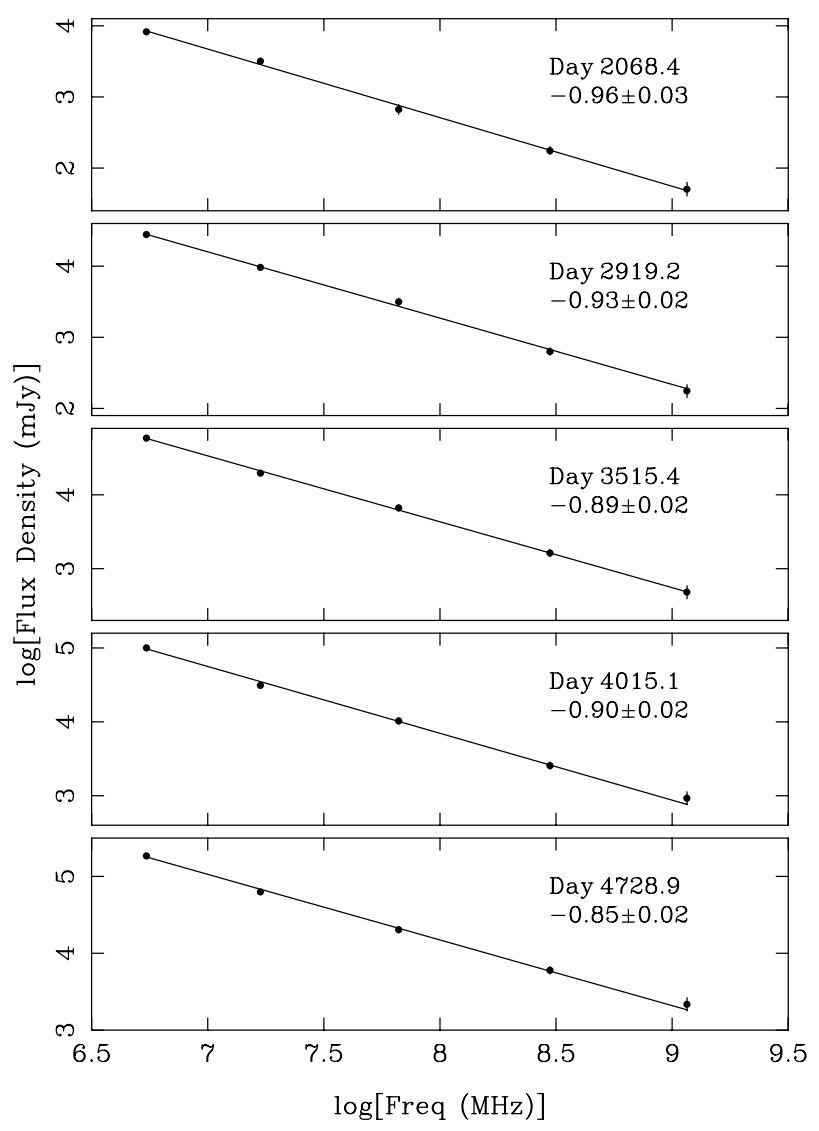

Figure 4 The radio spectrum of SNR 1987A at five different epochs.

Figure 4 shows MOST flux densities from Ball et al. (2001) and from the ATCA at 1.4, 2.4, 4.8, and $8.6 \mathrm{GHz}$ at five epochs spread through the data set. Values of the spectral index $\alpha$, where $S=v^{\alpha}$ and $v$ is the frequency, found by linear regression, are given on each plot. Quoted errors are $1 \sigma$.

To test for systematic curvature in the spectrum, the spectral indices were calculated using the three lowest frequencies, $\alpha_{1}$, and separately using the three highest frequencies, $\alpha_{2}$. If there is systematic curvature in the spectrum, then the difference $\alpha_{1}-\alpha_{2}$ should be significant, and roughly constant. In fact, the difference is 


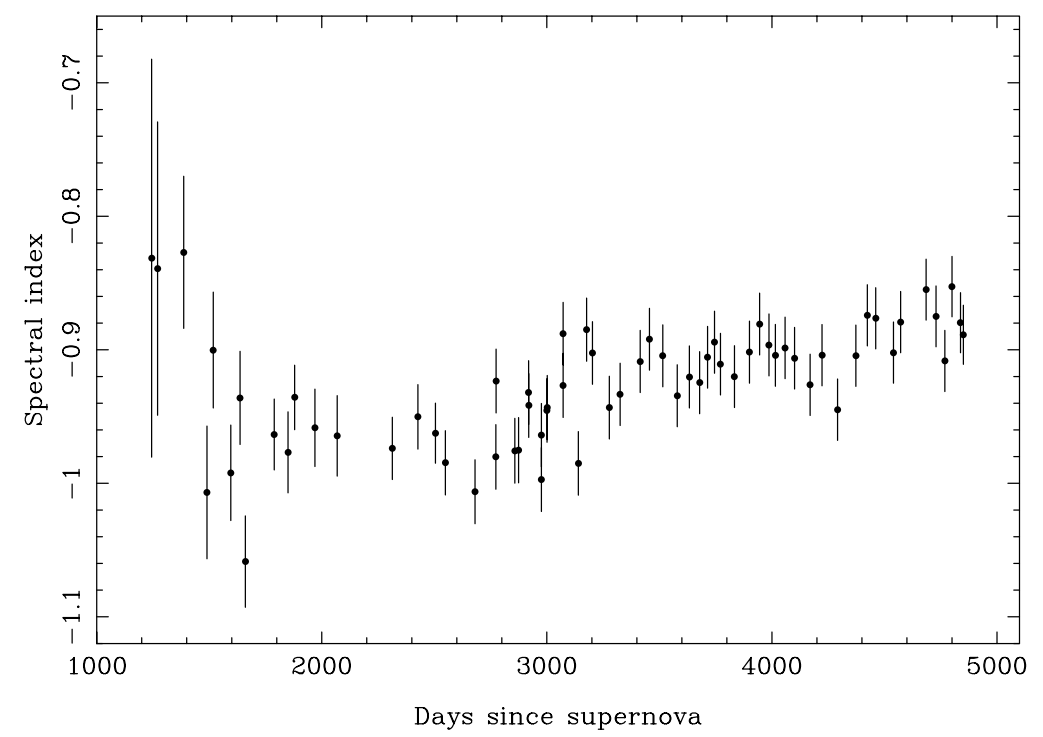

Figure 5 Spectral indices of the radio emission from SNR 1987A as a function of time obtained by combining the MOST $843 \mathrm{MHz}$ data of Ball et al. (2001) with ATCA data at 1.4, 2.4, 4.8, and 8.6 GHz.

typically small and of either sign, showing that there is no systematic curvature. The mean difference $\alpha_{1}-\alpha_{2}$ across the entire data set is -0.035 compared to the rms fluctuation of 0.187 .

The observation that the relative flux density gradient is both greater and increasing more rapidly at the higher frequencies (Table 1) implies a systematic change in the spectral index. Figure 5 shows the computed spectral indices from $843 \mathrm{MHz}$ to $8.4 \mathrm{GHz}$ as a function of time. Because of the uneven and non-simultaneous sampling at the different frequencies, spline curves were fitted to all data sets except at $1.4 \mathrm{GHz}$. Spectral indices were then computed by interpolating values at $843 \mathrm{MHz}, 2.4,4.8$, and $8.6 \mathrm{GHz}$ to times when the $1.4 \mathrm{GHz}$ flux density was measured, and fitting power-law spectra to flux densities. The interpolation was unreliable near the start of the data set for 2.4 and $8.6 \mathrm{GHz}$ because of sparse data, so only three points were fitted there.

Apart from a few high points between days 1200 and 1400 near the start of the data set (which have large error bars), there is a more or less steady increase in spectral index, corresponding to a flattening of the radio spectrum, throughout the whole data set. It is possible that the variation consists of step changes, with the most obvious step times being around day 3000 and day 4700. At early times, the mean spectral index was about -0.97 , but in the last year (2000) it was -0.88 .

The radio spectra are remarkably close to a power law and show no sign of either positive curvature, as would be expected if either free-free absorption or synchrotron self-absorption were important, or negative curvature as is predicted by diffusive shock acceleration theories (e.g. Reynolds \& Ellison 1992). The radio spectral index of about -0.9 is considerably steeper than the canonical -0.5 expected for synchrotron emission from relativistic electrons accelerated in strong shocks, for which the spectral index $\alpha=(1-s) / 2$ and the electron energy index $s=(r+2) /(r-2)$, where $r$ is the compression ratio in the shock (Jones \& Ellison 1991). For high Mach number shocks in a monatomic non-relativistic gas, $r=4.0$, giving $s=2.0$ and $\alpha=-0.5$.

A steeper radio spectrum implies a steeper electron energy distribution and a smaller compression ratio in the shock; $s \sim 2.8$ and $r \sim 2.7$ for $\alpha=-0.9$. Monte Carlo modelling of non-linear diffusive shock acceleration, including the dynamical effects of the accelerated particles (e.g. Baring et al. 1999), suggest that young and hence fast shocks have a larger compression ratio than older shocks, resulting in a flatter synchrotron spectrum. Duffy et al. (1995) included the effect of accelerated ions on the shock structure and were able to model the observed spectrum with the shock expanding into a terminated stellar wind structure. However, this model predicts a declining brightness and a steepening spectrum for the radio emission, both of which are contrary to observation.

The observed spectral index is also steeper than those for typical supernova remnants; no SNR in the Green (2000) catalogue has a spectral index definitely steeper than -0.8 and the mean spectral index for shell-type remnants is -0.51 . It is interesting to speculate that the current flattening of the spectrum represents an evolution toward the typical index of -0.5 . At the current rate, it will only take about 50 years to reach -0.5 .

The clump interaction model of Ball \& Kirk (1992) predicts a declining flux density at later times. However, this model was based on only two clumps. The model could be extended to invoke interaction with an increasing number of clumps, maybe tens or hundreds at the present time. A more accurate description would involve a statistical hierarchy of effective clump sizes. This could account for both the gradient increase and the short-term fluctuations in the observed flux density. Both radio and optical evidence (e.g. Spyromilio, Stathakis, \& Meurer 1993; 
Table 2. $9 \mathrm{GHz}$ ATCA observations of SNR 1987A used for imaging. The radius listed is that obtained by fitting a thin spherical shell to each $u-v$ data-set (see text)

\begin{tabular}{|c|c|c|c|c|c|c|}
\hline Mean epoch & $\begin{array}{l}\text { Observing } \\
\text { date }\end{array}$ & $\begin{array}{c}\text { Day } \\
\text { number }\end{array}$ & Array & $\begin{array}{l}\text { Frequencies } \\
(\mathrm{MHz})\end{array}$ & $\begin{array}{l}\text { Time on } \\
\text { source }(\mathrm{h})\end{array}$ & $\begin{array}{l}\text { Radius } \\
\left({ }^{\prime \prime}\right)\end{array}$ \\
\hline 1992.9 & $\begin{array}{l}1992 \text { Oct } 21 \\
1993 \text { Jan } 04 \\
1993 \text { Jan } 05\end{array}$ & $\begin{array}{l}2068 \\
2142 \\
2143\end{array}$ & $\begin{array}{l}6 \mathrm{C} \\
6 \mathrm{~A} \\
6 \mathrm{~A}\end{array}$ & $\begin{array}{l}8640,8900 \\
8640,8900 \\
8640,8900\end{array}$ & $\begin{array}{r}15 \\
13 \\
5\end{array}$ & $\begin{array}{l}0.66(2) \\
0.66(2) \\
0.62(2)\end{array}$ \\
\hline 1993.6 & $\begin{array}{l}1993 \text { Jun } 24 \\
1993 \text { Jul } 01 \\
1993 \text { Oct } 15\end{array}$ & $\begin{array}{l}2314 \\
2321 \\
2426\end{array}$ & $\begin{array}{l}6 \mathrm{C} \\
6 \mathrm{C} \\
6 \mathrm{~A}\end{array}$ & $\begin{array}{l}8640,8900 \\
8640,8900 \\
8640,9024\end{array}$ & $\begin{array}{r}9 \\
10 \\
18\end{array}$ & $\begin{array}{l}0.62(1) \\
0.68(1) \\
0.69(1)\end{array}$ \\
\hline 1994.4 & $\begin{array}{l}\text { 1994 Feb } 16 \\
\text { 1994 Jun } 27 \\
\text { 1994 Jul } 01\end{array}$ & $\begin{array}{l}2550 \\
2683 \\
2687\end{array}$ & $\begin{array}{l}6 \mathrm{~B} \\
6 \mathrm{C} \\
6 \mathrm{~A}\end{array}$ & $\begin{array}{l}8640,9024 \\
8640,9024 \\
8640,9024\end{array}$ & $\begin{array}{r}9 \\
21 \\
10\end{array}$ & $\begin{array}{l}0.69(2) \\
0.659(7) \\
0.659(9)\end{array}$ \\
\hline 1995.7 & $\begin{array}{l}1995 \text { Jul } 24 \\
1995 \text { Aug } 29 \\
1995 \text { Nov } 06\end{array}$ & $\begin{array}{l}3074 \\
3111 \\
3178\end{array}$ & $\begin{array}{l}6 \mathrm{C} \\
6 \mathrm{D} \\
6 \mathrm{~A}\end{array}$ & $\begin{array}{l}8640,9024 \\
8896,9152 \\
8640,9024\end{array}$ & $\begin{array}{l}7 \\
7 \\
9\end{array}$ & $\begin{array}{l}0.687(8) \\
0.69(2) \\
0.685(6)\end{array}$ \\
\hline 1996.7 & $\begin{array}{l}1996 \text { Jul } 21 \\
\text { 1996 Sep } 08 \\
1996 \text { Oct } 05\end{array}$ & $\begin{array}{l}3437 \\
3486 \\
3512\end{array}$ & $\begin{array}{l}6 \mathrm{C} \\
6 \mathrm{~B} \\
6 \mathrm{~A}\end{array}$ & $\begin{array}{l}8640,9024 \\
8640,9024 \\
8896,9152\end{array}$ & $\begin{array}{r}14 \\
13 \\
8\end{array}$ & $\begin{array}{l}0.688(4) \\
0.684(4) \\
0.692(6)\end{array}$ \\
\hline 1998.0 & $\begin{array}{l}1997 \text { Nov } 11 \\
1998 \text { Feb } 18 \\
1998 \text { Feb } 21\end{array}$ & $\begin{array}{l}3914 \\
4013 \\
4016\end{array}$ & $\begin{array}{l}6 \mathrm{C} \\
6 \mathrm{~A} \\
6 \mathrm{~B}\end{array}$ & $\begin{array}{l}8512,8896 \\
8896,9152 \\
9024,8512\end{array}$ & $\begin{array}{r}19 \\
15 \\
7\end{array}$ & $\begin{array}{l}0.715(5) \\
0.733(5) \\
0.735(4)\end{array}$ \\
\hline 1998.9 & $\begin{array}{l}1998 \text { Sep } 13 \\
1998 \text { Oct } 31 \\
1999 \text { Feb } 12\end{array}$ & $\begin{array}{l}4220 \\
4268 \\
4372\end{array}$ & $\begin{array}{l}6 \mathrm{~A} \\
6 \mathrm{D} \\
6 \mathrm{C}\end{array}$ & $\begin{array}{l}8896,9152 \\
9024,8512 \\
8512,8896\end{array}$ & $\begin{array}{l}12 \\
11 \\
10\end{array}$ & $\begin{array}{l}0.721(3) \\
0.729(5) \\
0.737(3)\end{array}$ \\
\hline 1999.7 & $\begin{array}{l}1999 \text { Sep } 05 \\
1999 \text { Sep } 12\end{array}$ & $\begin{array}{l}4578 \\
4585\end{array}$ & $\begin{array}{l}6 \mathrm{D} \\
6 \mathrm{~A}\end{array}$ & $\begin{array}{l}9152,8768 \\
8512,8896\end{array}$ & $\begin{array}{l}11 \\
14\end{array}$ & $\begin{array}{l}0.754(4) \\
0.736(4)\end{array}$ \\
\hline 2000.8 & $\begin{array}{l}2000 \text { Sep } 28 \\
2000 \text { Nov } 12\end{array}$ & $\begin{array}{l}4966 \\
5011\end{array}$ & $\begin{array}{l}6 \mathrm{~A} \\
6 \mathrm{C}\end{array}$ & $\begin{array}{l}8512,8896 \\
8512,8896\end{array}$ & $\begin{array}{l}10 \\
11\end{array}$ & $\begin{array}{l}0.756(2) \\
0.764(3)\end{array}$ \\
\hline
\end{tabular}

van Dyk et al. 1994; Spyromilio 1994) point to a clumpy circumstellar medium around other supernovae.

\section{Imaging Observations of SNR 1987A}

\subsection{Resolved Radio Images of SNR 1987A}

In previous papers we have shown that the ATCA's diffraction-limited resolution at $9 \mathrm{GHz}$ of 0.9 is sufficient to resolve the radio emission from SNR 1987A (StaveleySmith et al. 1993b) and that super-resolution techniques can be used to improve the resolution to $\sim 0^{\prime \prime} .5$. At this resolution, the radio emission forms a limb-brightened shell, with brightness enhancements on the eastern and western sides (Staveley-Smith et al. 1993a; Briggs 1994; Gaensler et al. 1997).

We have continued to make regular observations of SNR 1987A at $9 \mathrm{GHz}$; observing parameters for all imaging observations are given in Table 2 . We have analysed these data in the same manner as described by Gaensler et al. (1997), forming an image at each epoch and deconvolving the resulting image using a maximum entropy algorithm. From 1996 onwards, the source has been of sufficient signal-to-noise that phase self-calibration can be successfully applied to the data, resulting in a significant improvement in the accuracy of the complex gains for each antenna.
The resulting series of images are shown in Figures 6 and 7 under the conditions of diffraction-limited resolution and super-resolution, respectively. In these figures and Figure 9, the RA and Dec offsets are with respect to J2000 RA $05^{\mathrm{h}} 35^{\mathrm{m}} 28^{\mathrm{s}} .00$, Dec $-69^{\circ} 16^{\prime} 11^{\prime \prime} .1$ The diffractionlimited images indicate that the source is clearly extended, primarily in the east-west direction, and continues to brighten. In the super-resolved sequence of images, it can be seen that the shell-like morphology reported by Staveley-Smith et al. (1993a) and by Gaensler et al. (1997) is maintained throughout, with two bright regions on the east and west sides of the rim.

\subsection{Model Fits to the Radio Morphology}

Gaensler et al. (1997) showed that the size of SNR 1987A could be quantified at each epoch by approximating the morphology of SNR 1987A by a thin spherical shell of arbitrary position, flux, and radius. The best-fit parameters are found by computing the Fourier transform of this shell, subtracting this transform from the $u-v$ data, and then adjusting the properties of the model until the corresponding $\chi^{2}$ parameter is minimised (see also Staveley-Smith et al. 1993b). Using such an approach, Gaensler et al. (1997) were able to show that the radius of the supernova remnant increased from 0".65 at epoch 1992.9 to 0".68 at epoch 1995.7, corresponding to a (surprisingly low) 


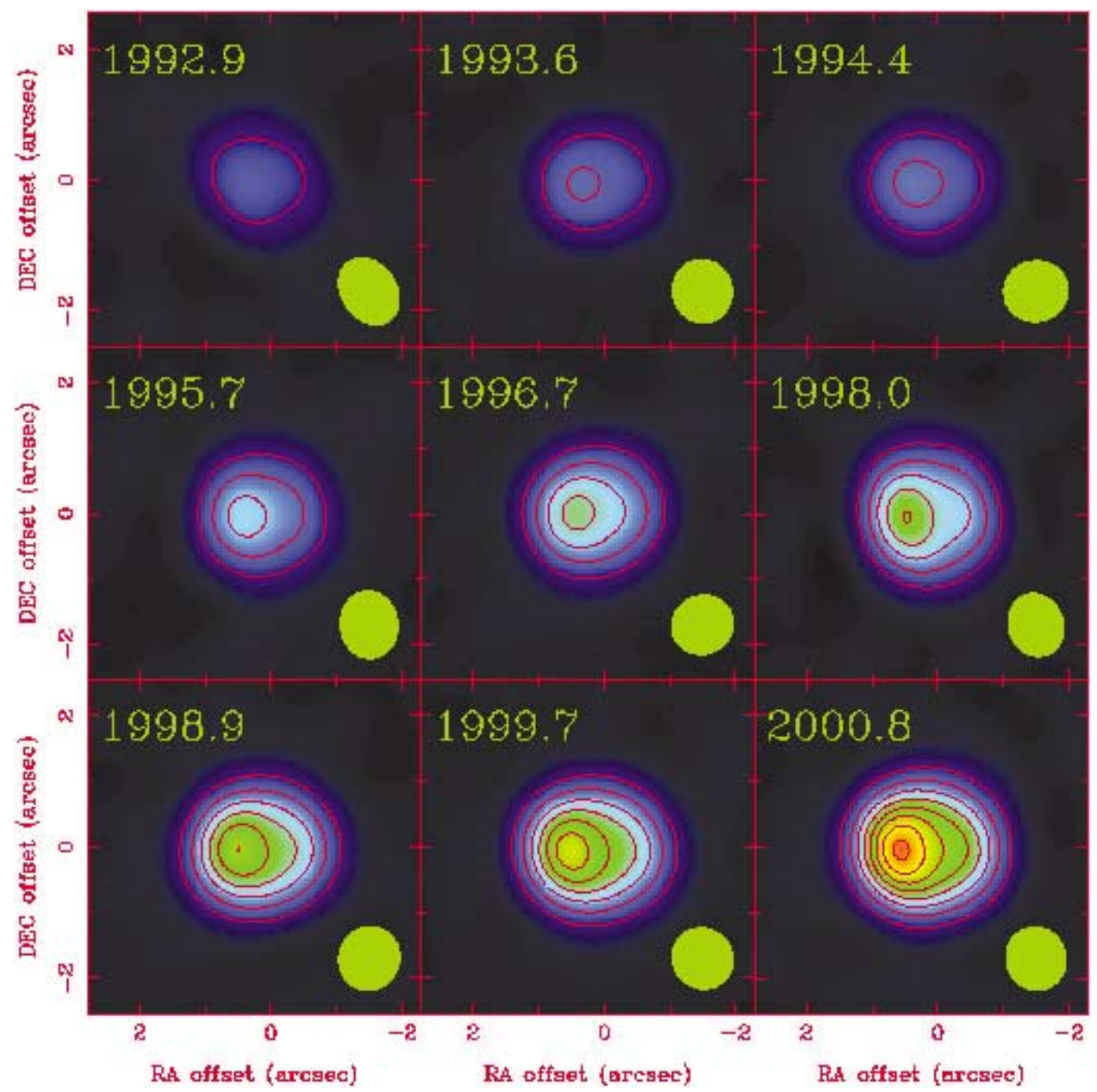

Figure 6 Diffraction-limited $9 \mathrm{GHz}$ images of SNR 1987A at the nine epochs listed in Table 2. The false-colour range in each panel is -0.1 to $+16.0 \mathrm{mJy}_{\text {beam }}^{-1}$, while contours are at levels of $1.5,3.0,4.5, \ldots, 13.5 \mathrm{mJy}_{\text {beam }}{ }^{-1}$. The FWHM dimensions of the diffraction-limited synthesised beam for each epoch are shown at the lower right of each panel.

mean expansion velocity of $2800 \pm 400 \mathrm{~km} \mathrm{~s}^{-1}$ (assuming a distance to the supernova of $50 \mathrm{kpc}$ ).

Here we extend and expand on these attempts to quantify the changes in the radio remnant. We first fit a spherical shell to all subsequent data sets. The resulting radii are listed in Table 2, where the uncertainty in the last quoted digit is given in parentheses. A fit to these radii gives a linear expansion rate of $3500 \pm 100 \mathrm{~km} \mathrm{~s}^{-1}$. The mean radius at each observing epoch is listed in Table 3 and plotted in Figure 8.

The signal-to-noise of the data presented by Gaensler et al. (1997) was too low to justify more complex fits to the data. However, we are now in a position to compare a thin spherical shell to other models. We first note that other simple spherically symmetric models, such as faceon rings and Gaussians, produce significant residuals, and are clearly inconsistent with the data. In Figure 9 we show three possible fits to the data from epoch 1998.0. In each case, we have generated an input model (shown in the first column). We have then simulated an observation of the sky distribution corresponding to this model as follows: we Fourier transform the model, then multiply this transform by the transfer function of the ATCA observations from this epoch to produce a set of $u-v$ tracks identical to those of the real data. We then image these visibilities in the same way as for the real data, and similarly deconvolve and super-resolve the image, to give the result shown in the second column. We also subtract the $u-v$ data corresponding to the model from the observed data, and then image the resulting visibilities to produce the residual image shown in the third column.

In the first row of Figure 9, we show the results of fitting a thin spherical shell to the 1998.0 data (cf. Gaensler et al. 1997) - the corresponding best-fit flux and radius are $18.4 \mathrm{mJy}$ and 0.73 . The resulting image successfully approximates the limb brightened morphology seen in Figure 7, but lacks the enhancements in brightness seen on either side of the shell. The residual image, while having small amplitudes in an absolute sense, shows clear and systematic differences between this simple model and the data (maximum fractional residual of $\sim 20 \%$ ).

Given that the main difference between a thin shell model and the data seems to be the presence of the bright lobes on either side, we next generate a model involving a thin shell (of arbitrary flux, position, and radius), and two point sources of emission (each of arbitrary flux and 


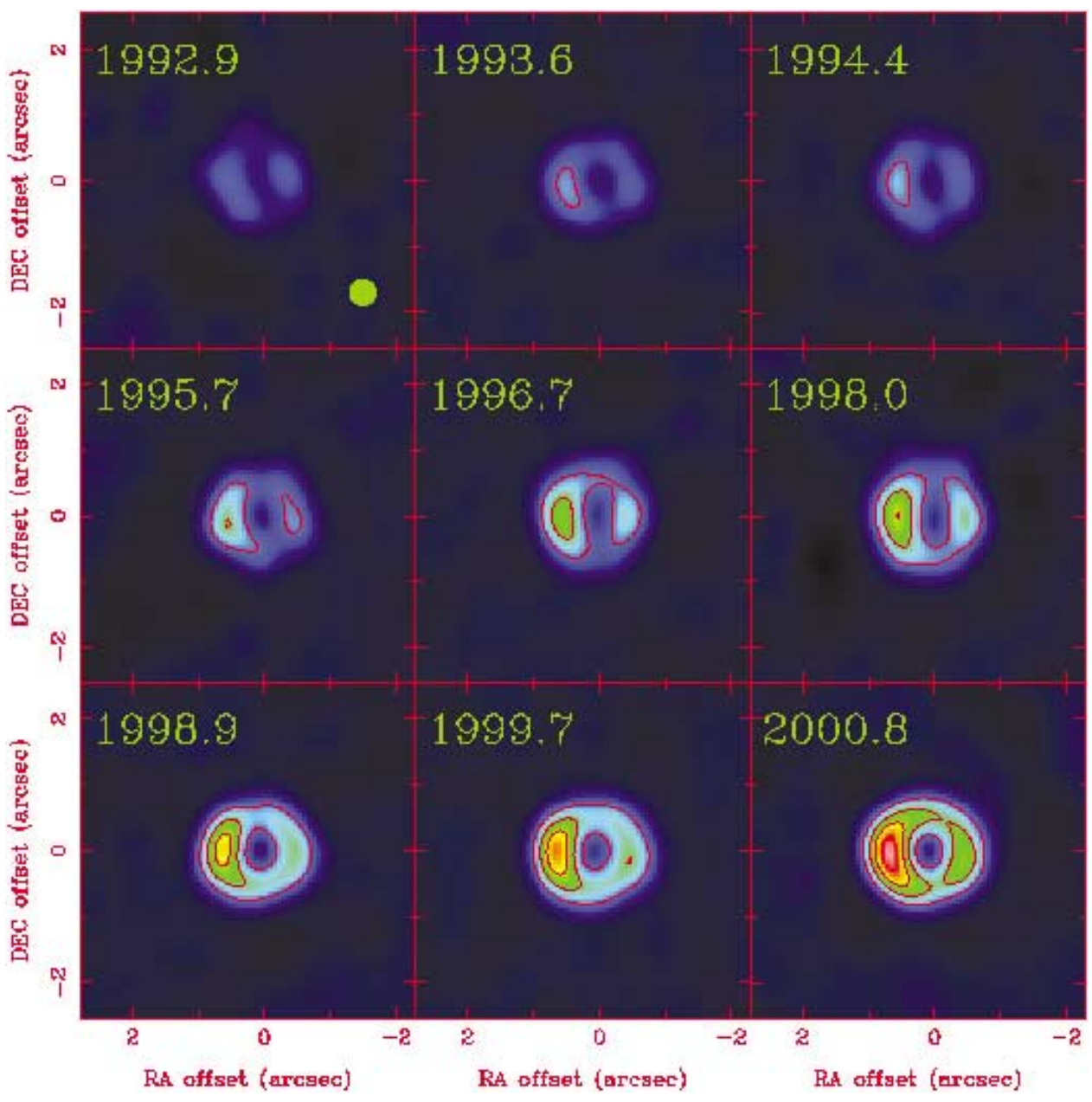

Figure 7 Super-resolved $9 \mathrm{GHz}$ images of SNR 1987A at the nine epochs listed in Table 2. The false-colour range in each panel is -0.1 to $+5.0 \mathrm{mJy}_{\text {beam }}^{-1}$, while contours are at levels of $0.4,0.8,1.2, \ldots, 2.8 \mathrm{mJy}$ beam $^{-1}$. The FWHM dimensions of the super-resolved synthesised beam are shown at the lower-right of the first panel.

Table 3. Parameters from model fits to the $9 \mathrm{GHz}$ images

\begin{tabular}{|c|c|c|c|c|c|c|c|c|c|c|c|}
\hline \multirow{2}{*}{$\begin{array}{l}\text { Mean } \\
\text { epoch }\end{array}$} & \multirow{2}{*}{$\begin{array}{c}\text { Total flux } \\
\text { density } \\
(\mathrm{m} J y)\end{array}$} & \multicolumn{4}{|c|}{ Fit to shell alone } & \multicolumn{6}{|c|}{ Fit to shell +2 points } \\
\hline & & $\begin{array}{c}S_{\text {shell }} \\
(\mathrm{m} \mathrm{Jy})\end{array}$ & $\begin{array}{c}r_{\text {shell }} \\
\left({ }^{\prime \prime}\right)\end{array}$ & $\begin{array}{c}S_{\text {shell }} \\
\text { (m Jy) }\end{array}$ & $\begin{array}{c}r_{\text {shell }} \\
\left({ }^{\prime \prime}\right)\end{array}$ & $\begin{array}{c}S_{1} \\
(\mathrm{~m} \mathrm{Jy})\end{array}$ & $\begin{array}{c}\delta \mathrm{RA}_{1} \\
\left(^{\prime \prime}\right)\end{array}$ & $\begin{array}{c}\delta \operatorname{Dec}_{1} \\
\left({ }^{\prime \prime}\right)\end{array}$ & $\begin{array}{c}S_{2} \\
(\mathrm{mJy})\end{array}$ & $\begin{array}{c}\delta \mathrm{RA}_{2} \\
\left({ }^{\prime \prime}\right)\end{array}$ & $\begin{array}{c}\delta \operatorname{Dec}_{2} \\
\left({ }^{\prime \prime}\right)\end{array}$ \\
\hline 1992.9 & $5.6(2)$ & 5.2 & $0.668(9)$ & & & & & & & & \\
\hline 1993.6 & $6.9(2)$ & 6.6 & $0.659(6)$ & 5.1 & $0.73(2)$ & 1.2 & $0.26(3)$ & $-0.05(2)$ & 0.4 & $-0.73(9)$ & $0.06(5)$ \\
\hline 1994.4 & $7.5(1)$ & 7.8 & $0.671(4)$ & 6.2 & $0.75(2)$ & 1.3 & $0.27(2)$ & $-0.03(1)$ & 0.4 & $-0.69(7)$ & $0.06(4)$ \\
\hline 1995.7 & $11.8(1)$ & 11.0 & 0.684 (4) & 8.6 & $0.71(2)$ & 1.9 & $0.35(3)$ & $-0.03(1)$ & 0.5 & $-0.76(8)$ & $0.04(5)$ \\
\hline 1996.7 & $15.5(1)$ & 15.1 & $0.688(2)$ & 12.4 & $0.764(7)$ & 2.5 & $0.32(1)$ & $0.03(1)$ & 0.4 & $-0.69(6)$ & $0.15(3)$ \\
\hline 1998.0 & $18.3(1)$ & 18.4 & $0.731(2)$ & 15.0 & $0.786(8)$ & 3.1 & $0.34(1)$ & $-0.02(1)$ & 0.4 & $-0.75(7)$ & $-0.02(5)$ \\
\hline 1998.9 & $21.7(1)$ & 21.5 & $0.730(2)$ & 17.2 & $0.778(6)$ & 3.4 & $0.40(1)$ & $-0.02(1)$ & 1.0 & $-0.81(3)$ & $-0.04(2)$ \\
\hline 1999.7 & $24.7(1)$ & 24.4 & $0.742(2)$ & 19.7 & $0.796(6)$ & 3.9 & $0.41(1)$ & $-0.02(1)$ & 1.0 & $-0.81(3)$ & $-0.05(2)$ \\
\hline 2000.8 & $30.8(1)$ & 30.8 & $0.761(2)$ & 24.9 & $0.804(4)$ & 4.8 & $0.46(1)$ & $-0.01(1)$ & 1.3 & $-0.82(2)$ & $-0.03(2)$ \\
\hline
\end{tabular}

position). For the 1998.0 data set, the best-fit parameters for this model are a shell of flux $15.0 \mathrm{mJy}$ and radius 0.79 , and point sources of fluxes 3.1 and $0.4 \mathrm{mJy}$, one projected near the edge of, but inside, the eastern half of the shell, and the other similarly positioned on the western half. Note that the radius of the shell in this fit is $8 \%$ larger than that obtained by fitting to a shell alone. The resulting model, image, and residual are all shown in the second row of Figure 9. The image corresponding to the model now reasonably approximates the morphology seen in the real data, and the residuals are greatly reduced (maximum fractional residual of $\sim 5 \%$ ) when compared to the case of a shell alone.

We can further improve the fit between the model and the data by increasing the number of point sources in the model. In the bottom row of Figure 9, we show a fit to the 


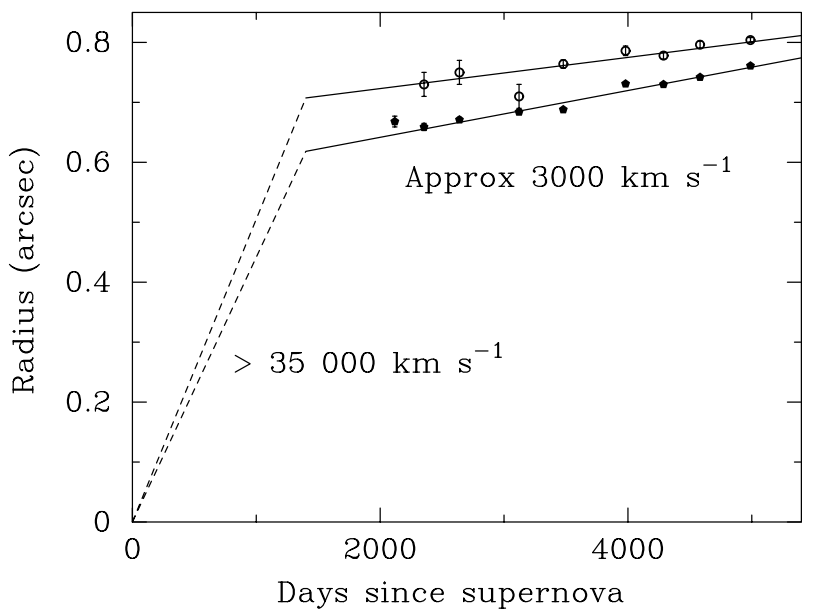

Figure 8 Inferred radius of the radio remnant SNR 1987A as a function of time. The filled points represent radii determined by fitting a thin spherical shell to the data, while open points correspond to a model involving a shell plus two point sources. The solid lines indicate the respective best-fit linear increases, while the dashed lines are extrapolations between the epoch of the supernova explosion and the time when radio emission re-appeared.
1998.0 data involving a spherical shell plus seven point sources. The large number of free parameters (25 in this case) makes finding an absolute minimum in $\chi^{2}$ very difficult - thus the 'best fit' we have shown was found by trial and error. The shell has flux density $11.4 \mathrm{mJy}$ and radius 0.72 ( $2 \%$ smaller than in the case of a shell alone); the point sources range in flux density between 0.3 and $3.5 \mathrm{mJy}$, and are distributed around the perimeter of the shell. The model image and residual image both indicate a very good match between the model and the data (maximum fractional residual $\sim 2 \%$ ). We emphasise, though, that we make no claims to uniqueness for this solution.

Given the difficulty in finding a multiple component fit to the data by trial and error, and the non-uniqueness of this solution, we do not here present multi-parameter fits to other epochs such as those shown in the third row of Figure 9. However, it seems clear that a shell alone is no longer the best simple model of the data, and that a shell with two point sources is a significant improvement. We therefore have fitted all epochs with these two models, with all parameters free to vary in each case. The results

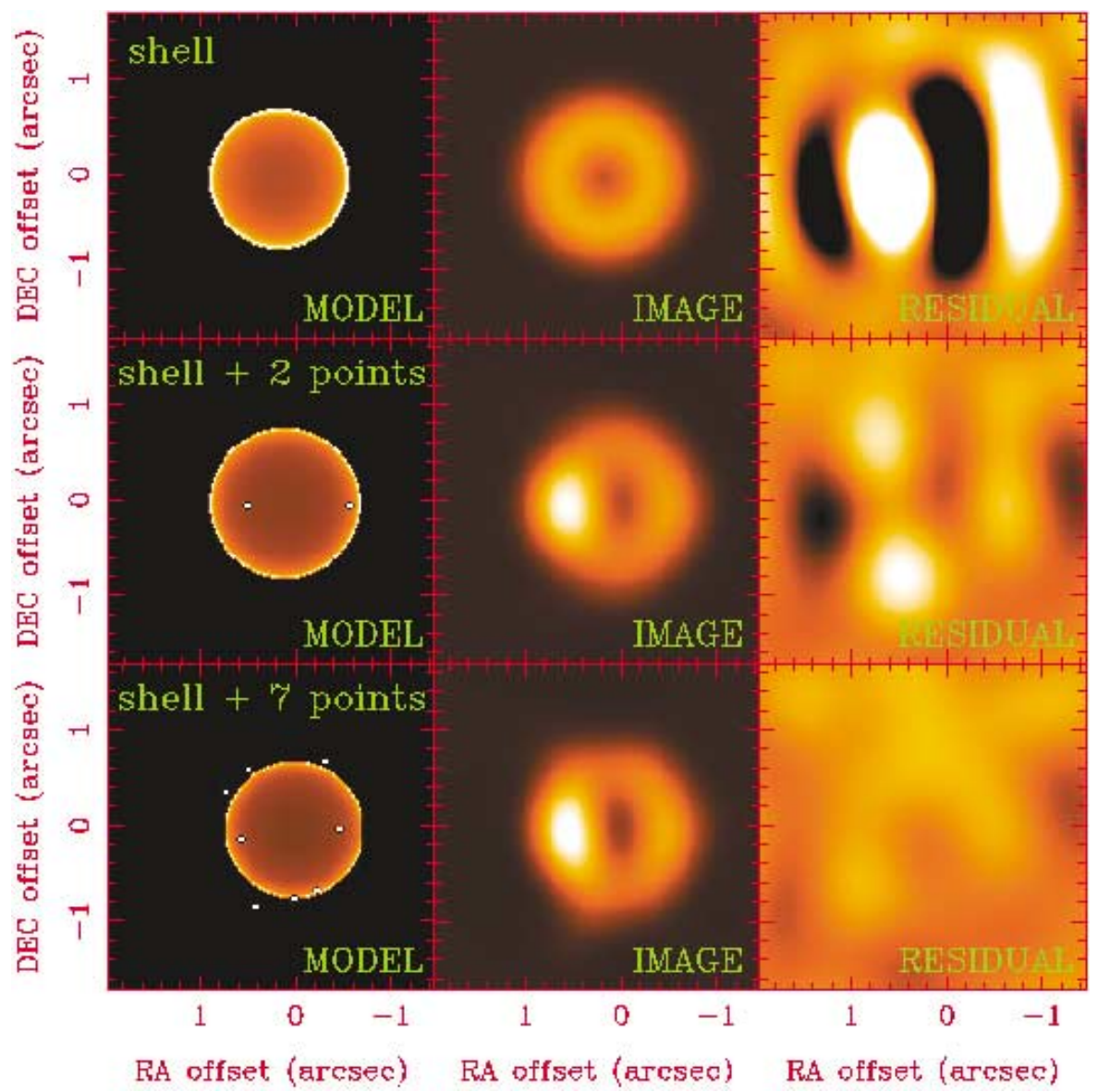

Figure 9 Models of the radio emission from SNR 1987A. Each row represents a different attempt to model the radio emission from epoch 1998.0: a thin spherical shell; a thin shell plus two hot spots; a thin shell plus seven hot spots. The first column shows the input image for each model, using a false-colour range of $0-0.01 \mathrm{mJy}_{\text {beam }}^{-1}$; the second column shows the results of a simulated super-resolved observation of the input image, using a greyscale range of -0.16 to $+3.05 \mathrm{mJy} \mathrm{beam}^{-1}$; the third column shows an image of the residuals remaining once the model has been subtracted from the original data, using a false-colour range -0.2 to $+0.3 \mathrm{mJy}_{\text {beam }}{ }^{-1}$. In the third model, the east point source within the shell has a flux density of $3.5 \mathrm{mJy}$; the other six have flux densities in the range $0.3-0.8 \mathrm{mJy}$. 
of these fits are listed in Table 3 and plotted in Figure 8. Offsets of the two point sources in Table 3 are with respect to the reference position used for Figures 6, 7, and 9. The data from epoch 1992.9 are of low signal-to-noise, and no good fit using this more complex model could be found.

It can be seen from Table 3 that the radius of the shell in models which include two point sources is $5-10 \%$ larger than that obtained in the shell-only model used by Gaensler et al. (1997). It is also clear that the point sources are moving outward with the expansion of the shell, the eastern one apparently at a larger relative rate than the shell. As for the shell model, we can fit these larger radii by a linear increase, to obtain an expansion speed of $2300 \pm 300 \mathrm{~km} \mathrm{~s}^{-1}$, about $35 \%$ slower than in the case for the shell alone. However, we note that if multiparameter fits like those in the third row of Figure 9 are carried out for other epochs, the resulting radii are $\sim 5 \%$ smaller than for the fits to a shell alone, and the resulting inferred expansion speed is $\sim 3700 \mathrm{~km} \mathrm{~s}^{-1}$. While we have not carried out the corresponding analysis here, we note that Staveley-Smith et al. (1993a) fitted the radio emission from SNR 1987A with a thick spherical shell of outerto-inner radius ratio 1.25 . This results in a shell diameter about $10 \%$ greater than for the thin shell fit, consistent with the range of possible radii considered here.

We therefore conclude that the radius of the remnant as determined from fitting to the $u-v$ data is uncertain by $\sim 10 \%$, and that the resulting expansion velocity is uncertain by $\sim 30 \%$. The main conclusions from earlier results - that the radio emission is originating from a region within the equatorial ring, and that the material producing this emission was initially moving rapidly but now has a very low rate of expansion - are unchanged, even when the assumption of spherical symmetry is relaxed.

\subsection{Comparison with Other Wavelengths and Discussion}

In Figure 10 we compare our $9 \mathrm{GHz}$ ATCA data to recent observations with $H S T$ and with Chandra. In the upper

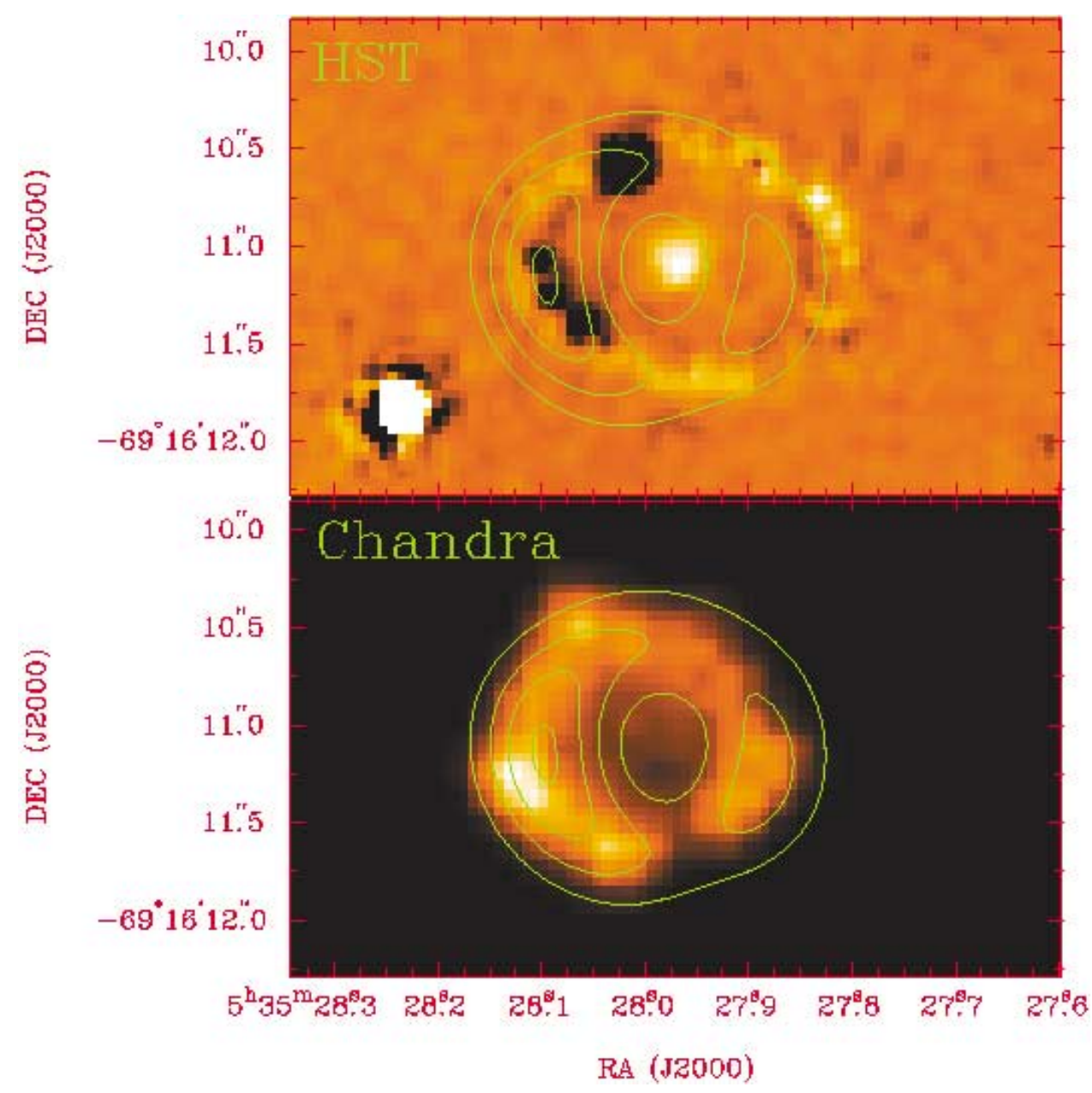

Figure 10 Multi-wavelength comparisons of SNR 1987A. The upper panel overlays HST and ATCA observations of SNR 1987A: the false-colour image represents the difference of HST WFPC2 images in the F6565N filter, obtained by subtracting an image taken in 1998 February from a similar image taken in 1999 April (Lawrence et al. 2000), while the contours correspond to the super-resolved 2000.8-epoch ATCA image, using contour levels of 1.1, 2.2, 3.3, and $4.4 \mathrm{mJy}$ beam $^{-1}$. The lower panel overlays Chandra and ATCA observations - the false-colour represents a super-resolved Chandra ACIS-S image covering the energy range $0.3-8 \mathrm{keV}$ at epoch 1999.8 (Burrows et al. 2000), while the contours indicate radio data as in the upper panel. 
panel, the super-resolved ATCA image from epoch 2000.8 is compared to the difference image of optical emission around the supernova produced by Lawrence et al. (2000). The HST image has been registered on the radio frame with an accuracy of better than $0{ }^{\prime \prime} 1$ using the position of the central star from Reynolds et al. (1995). It shows both the fading equatorial ring, and at least seven hot spots on this ring, all brightening as the supernova shock begins to interact with dense circumstellar material. It can be seen from this optical/radio comparison that the conclusions made by Gaensler et al. (1997) are maintained: the bright radio lobes align with the major axis of the optical ring, with the brighter eastern lobe clearly more distant from the central star than the fainter western lobe. Within the uncertainties, the eastern radio lobe is coincident with some of the optical hot spots (but not the brighest one), just inside the optical ring. In the west, the optical emission lies outside the radio lobe, close to the lowest radio contour in Figure 10. In fact, there is a good correspondence of the radio emission with optical emission within the optical ring (not visible in Figure 10) corresponding to the reverse shock, as reported by Garnavich, Kirshner, \& Challis (1999). Just as in the radio data, two optical lobes are seen, one to the east and one to the west of the supernova site. Also similar to the case in the radio, the eastern optical lobe is brighter than and further from the supernova than the western lobe.

The lower panel of Figure 10 compares radio emission with a super-resolved image obtained by Chandra at epoch 1999.8 (Burrows et al. 2000). The X-ray and radio emission from the supernova remnant both take the form of limb brightened shells; the images were aligned by placing the estimated centre of the X-ray remnant on the Reynolds et al. (1995) position (D. Burrows 2001, private communication) and hence is less accurate than the optical-radio alignment. While there is a good correspondence between the brightest regions in each waveband, the X-ray maxima appear to lie outside the radio maxima. This is perhaps surprising since theoretical models (e.g. Borkowski, Blondin, \& McCray 1997) suggest that the radio emission is generated just inside the outer shock whereas the X-ray emission is generated by a reverse shock compressing and heating the denser ejecta as it propagates inwards. It is worth noting, however, that X-ray emission lying outside the radio emission and interpreted as coming from the outer shock has been detected in the SNR 1E 0102.2-7219 by Gaetz et al. (2000).

The two-lobed radio morphology seen for SNR 1987A was apparent at least as early as 1992 (Figure 7; StaveleySmith et al. 1993a), while optical hot spots did not begin to appear on the eastern side of the optical ring until 1995 and on the western side until 1998 (Lawrence et al. 2000). Furthermore, the earliest appearing and brightest optical hot spot does not coincide in position angle with either of the two main radio lobes, nor with the brightest X-ray emission seen by Chandra (Figure 10; Burrows et al. 2000). We therefore argue that the more rapid rate in the increase of radio emission beginning around day 3000, as reported by Ball et al. (2001) and confirmed in Section 2 above, is not related to the appearance of optical hot spots seen at around the same time. While Ball et al. (2001) have pointed out that the various optical hot spots turned on at about the right time for them to have been produced by the arrival of the radio-producing $\operatorname{shock}(\mathrm{s})$ in these regions, it seems clear that this had little effect on the radio morphology of the remnant. The best explanation for deviations from spherical symmetry in the radio morphology of SNR 1987A still seems to be that they result simply from regions of enhanced emission in the equatorial plane of the progenitor system, where circumstellar gas is expected to be both densest and closest to the progenitor star (cf. Gaensler et al. 1997). The fact that at both radio and optical wavelengths the eastern lobe is both brighter and more distant from the explosion site than the western lobe may represent an asymmetry in the distribution of ejecta.

It is notable that the emission underlying the radio hot spots is well described by a spherical shell and that, in all of the models, the flux density of the shell dominates the total flux density. This is surprising given the strong equatorial enhancement evident in the optical data and implied for the circumstellar gas. One might expect eventually to see a faster expansion of the radio remnant in the polar directions (Blondin, Lundqvist, \& Chevalier 1996), but there is currently no evidence for this. Part of the radio emission from the lobes may be attributed to an equatorial enhancement but, since such an enhancement would be symmetric, it cannot account for all of the emission seen from the brighter eastern lobe. It is worth noting that the shell of SN 1993J also appears to be quite spherical (Marcaide et al. 1997; Bartel et al. 2000).

\section{Future Prospects}

Results at all wavelengths suggest that there is an increasing interaction between the expanding ejecta and the circumstellar material. Hot spots around or just inside the emission-line ring are certainly becoming more numerous and prominent in the optical band. Neither the radio imaging nor the X-ray imaging has sufficient resolution to separately identify hot spots. However, the modelling of the radio $u-v$ data and the possible short-term fluctuations in the rise of the high frequency radio flux densities suggest that compact radio hot spots do exist. The overall morphology and the time evolution of the radio emission suggest that there is no detailed correspondence of the radio hot spots with the hot spots seen in the optical data and, in fact, that the radio hot spots are much more numerous and widespread.

We have shown that the rate of increase of the radio emission from SNR 1987A has slowly increased over the past few years. It is possible that the rate of increase of the radio (and other) emission will dramatically increase when significant amounts of ejecta begin to interact with the dense circumstellar gas of the inner ring. Extrapolation of the radii and expansion speeds resulting from model fits to the radio data suggest that this will happen in $2004 \pm 2$. The ATCA is presently being upgraded for observations 
in the $12 \mathrm{~mm}$ and $3 \mathrm{~mm}$ bands, with an expected commissioning date of late-2002. While the radio remnant is unlikely to be detectable at $3 \mathrm{~mm}$, we expect $12 \mathrm{~mm}$ observations to provide increased resolution. Extrapolating the present flux density increase and the currently observed power-law spectrum, we expect a flux density at $20 \mathrm{GHz}$ in mid-2002 of about $17 \mathrm{mJy}$. The diffraction limited halfpower beamwidth at $20 \mathrm{GHz}$ will be about 0 .'4, somewhat less than the present super-resolved beamwidth at $9 \mathrm{GHz}$. Even in 2002, it is likely that super-resolution will be able to be applied to the $20 \mathrm{GHz}$ data, giving a resolution of about $0^{\prime \prime} .2$, and this will certainly be true at later epochs if the flux density continues to rise. We hope and expect that this will reveal further detail in the radio images, allowing interesting comparisons with images obtained at other wavelengths and giving further insight into the physics of the radio emission process.

\section{Acknowledgements}

We thank Lewis Ball for making available a prepublication file of the MOST flux densities for SNR 1987A and for comments on an earlier version of the paper, Dave Burrows for providing the Chandra data, and Ben Sugerman and Peter Garnavich for providing HST data. We also thank the referees for helpful suggestions. The Australia Telescope is funded by the Commonwealth of Australia for operation as a National Facility managed by CSIRO. BMG acknowledges the support of NASA through Hubble Fellowship grant HF-01107.0198A awarded by the Space Telescope Science Institute, which is operated by the Association of Universities for Research in Astronomy, Inc., for NASA under contract NAS 5-26555.

\section{References}

Ball, L., Campbell-Wilson, D., Crawford, D. F., \& Turtle, A. J. 1995, ApJ, 453, 864

Ball, L., Crawford, D. F., Hunstead, R. W., Klamer, I., \& McIntyre, V. J. 2001, ApJ, 549, 599

Ball, L., \& Kirk, J. G. 1992, ApJ, 396, L39

Baring, M. G., Ellison, D. C., Reynolds, S. P., Grenier, I. A., \& Goret, P. 1999, ApJ, 513, 311

Bartel, N., et al. 2000, Science, 287, 112

Blondin, J. M., Lundqvist, P., \& Chevalier, R. A. 1996, ApJ, 472,257

Borkowski, K. J., Blondin, J. M., \& McCray, R. 1997, ApJ, 476, L31

Briggs, D. S. 1994, in The Restoration of HST Images and Spectra II, eds R. J. Hanisch, \& R. L. White (Baltimore: Space Telescope Science Institute), 250

Burrows, C. J., et al. 1995, ApJ, 452, 680

Burrows, D. N., et al. 2000, ApJ, 543, L149

Chevalier, R. A. 1992, Nature, 355, 617

Chevalier, R. A., \& Dwarkadas, V. V. 1995, ApJ, 452, L45
Chevalier, R. A., \& Fransson, C. 1987, Nature, 328, 44

Crotts, A. P. S., Kunkel, W. E., \& Heathcote, S. R. 1995, ApJ, 438, 724

Duffy, P., Ball, L., \& Kirk, J. G. 1995, ApJ, 447, 364

Gaensler, B. M., Manchester, R. N., Staveley-Smith, L., Tzioumis, A. K., Reynolds, J. E., \& Kesteven, M. J. 1997, ApJ, 479,845

Gaetz, T. J., Butt, Y. M., Edgar, R. J., Eriksen, K. A., Plucinsky, P. P., Schlegel, E. M., \& Smith, R. K. 2000, ApJ, 534, L47

Garnavich, P., Kirshner, R., \& Challis, P. 1999, IAUC 7102

Gorenstein, P., Hughes, J. P., \& Tucker, W. H. 1994, ApJ, 420, L25

Green, D. A. 2000, A Catalogue of Galactic Supernova Remnants (Cambridge: Mullard Radio Astronomy Observatory, Cavendish Laboratory), http://www.mrao.cam.ac.uk/surveys/snrs/

Hasinger, G., Aschenbach, B., \& Trümper, J. 1996, A\&A, 312, L9

Jauncey, D. L., Kemball, A., Bartel, N., Shapiro, I. I., Whitney, A. R., Rogers, A. E. E., Preston, R. A., \& Clark, T. 1988, Nature, 334,412

Jones, F. C., \& Ellison, D. C. 1991, SSRv, 58, 259

Lawrence, S. S., Sugerman, B. E., Bouchet, P., Crotts, A. P. S., Uglesich, R., \& Heathcote, S. 2000, ApJ, 537, L123

Marcaide, J. E., et al. 1997, ApJ, 486, L31

Montes, M. J., van Dyk, S. D., Weiler, K. W., Sramek, R. A., \& Panagia, N. 1998, ApJ, 506, 874

Montes, M. J., Weiler, K. W., Van Dyk, S. D., Panagia, N., Lacey, C. K., Sramek, R. A., \& Park, R. 2000, ApJ, 532, 1124

Plait, P. C., Lundqvist, P., Chevalier, R. A., \& Kirshner, R. P. 1995, ApJ, 439, 730

Pun, C. S. J., Sonneborn, G., Bowers, C., Gull, T., Heap, S., Kimble, R., Maran, S., \& Woodgate, B. 1997, IAUC 6665

Reynolds, J. E., et al. 1995, A\&A, 304, 116

Reynolds, S. P., \& Ellison, D. C. 1992, ApJ, 399, L75

Spyromilio, J. 1994, MNRAS, 266, 61

Spyromilio, J., Stathakis, R. A., \& Meurer, G. R. 1993, MNRAS, 263,530

Staveley-Smith, L., et al. 1992, Nature, 355, 147

Staveley-Smith, L., Briggs, D. S., Rowe, A. C. H., Manchester, R. N., Reynolds, J. E., Tzioumis, A. K., \& Kesteven, M. J. 1993a, Nature, 366, 136

Staveley-Smith, L., Manchester, R. N., Kesteven, M. J., Tzioumis, A. K., \& Reynolds, J. E. 1993b, PASA, 10, 331

Storey, M. C., \& Manchester, R. N. 1987, Nature, 329, 421

Turtle, A. J., et al. 1987, Nature, 327, 38

van Dyk, S. D., Weiler, K. W., Sramek, R. A., Rupen, M. P., \& Panagia, N. 1994, ApJ, 432, L115

Weiler, K. W., van Dyk, S. D., Montes, M. J., Panagia, N., \& Sramek, R. A. 1998, ApJ, 500, 51

\section{Appendix}

Table 4 gives flux densities measured at the four ATCA frequencies over the day range 2000-5100 (MJD 48449-51949). These are calibrated relative to the primary flux calibrator, PKS B1934-638, using standard techniques. This table, also including flux density measurements for the nearby unresolved source J0536-6918 and more recent measurements, is available at http://www. atnf.csiro.au/research/pulsar/snr/sn1987a/ 
Table 4. ATCA flux density measurements for SNR 1987A

\begin{tabular}{|c|c|c|c|c|c|c|c|}
\hline \multicolumn{2}{|c|}{$1.4 \mathrm{GHz}$} & \multicolumn{2}{|c|}{$2.4 \mathrm{GHz}$} & \multicolumn{2}{|c|}{$4.8 \mathrm{GHz}$} & \multicolumn{2}{|c|}{$8.6 \mathrm{GHz}$} \\
\hline $\begin{array}{l}\text { Day } \\
\text { number }\end{array}$ & $\begin{array}{l}\text { Flux density } \\
\qquad(\mathrm{mJy})\end{array}$ & $\begin{array}{c}\text { Day } \\
\text { number }\end{array}$ & $\begin{array}{l}\text { Flux density } \\
\text { (mJy) }\end{array}$ & $\begin{array}{c}\text { Day } \\
\text { number }\end{array}$ & $\begin{array}{l}\text { Flux density } \\
\text { (mJy) }\end{array}$ & $\begin{array}{c}\text { Day } \\
\text { number }\end{array}$ & $\begin{array}{l}\text { Flux density } \\
\text { (mJy) }\end{array}$ \\
\hline 1243.9 & $0.00 \pm 1.20$ & 1198.9 & $0.00 \pm 1.00$ & 1243.6 & $0.00 \pm 0.25$ & 1388.1 & $1.33 \pm 0.26$ \\
\hline 1270.7 & $2.92 \pm 1.20$ & 1314.5 & $3.27 \pm 1.00$ & 1269.7 & $0.66 \pm 0.25$ & 1432.2 & $1.12 \pm 0.26$ \\
\hline 1386.4 & $4.87 \pm 1.21$ & 1386.0 & $3.45 \pm 1.01$ & 1305.5 & $1.15 \pm 0.25$ & 1500.5 & $1.74 \pm 0.26$ \\
\hline 1490.0 & $7.61 \pm 1.22$ & 1517.9 & $6.13 \pm 1.02$ & 1333.7 & $1.49 \pm 0.26$ & 1515.0 & $2.34 \pm 0.28$ \\
\hline 1517.9 & $8.29 \pm 1.23$ & 1517.7 & $6.20 \pm 1.02$ & 1366.3 & $1.53 \pm 0.26$ & 1583.8 & $2.62 \pm 0.28$ \\
\hline 1517.7 & $9.55 \pm 1.23$ & 1595.8 & $5.55 \pm 1.01$ & 1385.2 & $1.96 \pm 0.26$ & 1593.8 & $2.21 \pm 0.27$ \\
\hline 1595.8 & $10.15 \pm 1.24$ & 1636.7 & $8.71 \pm 1.03$ & 1401.4 & $2.17 \pm 0.26$ & 1662.5 & $2.92 \pm 0.29$ \\
\hline 1636.7 & $15.52 \pm 1.29$ & 1660.5 & $9.15 \pm 1.04$ & 1402.4 & $2.15 \pm 0.26$ & 1661.5 & $2.50 \pm 0.28$ \\
\hline 1660.5 & $15.52 \pm 1.29$ & 1788.8 & $10.67 \pm 1.05$ & 1403.4 & $2.25 \pm 0.27$ & 1786.6 & $3.92 \pm 0.32$ \\
\hline 1788.8 & $20.59 \pm 1.35$ & 1850.1 & $13.07 \pm 1.07$ & 1404.3 & $2.12 \pm 0.26$ & 1852.1 & $4.37 \pm 0.33$ \\
\hline 1850.1 & $24.80 \pm 1.41$ & 1879.2 & $15.03 \pm 1.10$ & 1405.3 & $2.13 \pm 0.26$ & 1878.2 & $4.14 \pm 0.32$ \\
\hline 1879.2 & $24.04 \pm 1.40$ & 1969.7 & $17.95 \pm 1.14$ & 1407.1 & $2.06 \pm 0.26$ & 1947.8 & $4.70 \pm 0.34$ \\
\hline 1969.7 & $27.51 \pm 1.46$ & 2067.7 & $19.00 \pm 1.15$ & 1408.3 & $2.57 \pm 0.27$ & 1969.5 & $4.93 \pm 0.35$ \\
\hline 2068.4 & $33.15 \pm 1.56$ & 2313.7 & $23.25 \pm 1.22$ & 1409.3 & $2.53 \pm 0.27$ & 1986.4 & $4.64 \pm 0.34$ \\
\hline 2313.7 & $38.87 \pm 1.67$ & 2427.3 & $23.37 \pm 1.22$ & 1410.3 & $2.66 \pm 0.27$ & 2003.4 & $5.09 \pm 0.36$ \\
\hline 2427.3 & $39.96 \pm 1.70$ & 2505.2 & $25.91 \pm 1.27$ & 1431.2 & $2.29 \pm 0.27$ & 2067.5 & $5.48 \pm 0.37$ \\
\hline 2505.2 & $43.23 \pm 1.77$ & 2549.2 & $26.56 \pm 1.28$ & 1446.2 & $2.63 \pm 0.27$ & 2092.4 & $5.92 \pm 0.39$ \\
\hline 2549.2 & $43.83 \pm 1.78$ & 2680.7 & $28.40 \pm 1.31$ & 1460.1 & $3.03 \pm 0.28$ & 2142.2 & $5.43 \pm 0.37$ \\
\hline 2680.7 & $46.20 \pm 1.83$ & 2774.5 & $29.92 \pm 1.34$ & 1516.9 & $2.83 \pm 0.27$ & 2195.7 & $4.20 \pm 0.33$ \\
\hline 2774.5 & $49.26 \pm 1.90$ & 2773.7 & $30.40 \pm 1.35$ & 1524.9 & $2.74 \pm 0.27$ & 2261.8 & $6.04 \pm 0.39$ \\
\hline 2773.7 & $48.60 \pm 1.89$ & 2826.7 & $28.40 \pm 1.31$ & 1586.8 & $2.97 \pm 0.28$ & 2299.9 & $7.14 \pm 0.44$ \\
\hline 2857.7 & $50.90 \pm 1.94$ & 2857.7 & $29.30 \pm 1.33$ & 1594.8 & $4.50 \pm 0.31$ & 2299.7 & $5.80 \pm 0.38$ \\
\hline 2874.3 & $48.40 \pm 1.88$ & 2874.3 & $25.20 \pm 1.25$ & 1635.6 & $5.31 \pm 0.33$ & 2313.7 & $6.92 \pm 0.43$ \\
\hline 2919.2 & $53.77 \pm 2.01$ & 2919.2 & $33.11 \pm 1.41$ & 1662.5 & $5.02 \pm 0.32$ & 2320.7 & $6.90 \pm 0.43$ \\
\hline 2919.3 & $51.38 \pm 1.95$ & 2919.3 & $34.20 \pm 1.43$ & 1746.8 & $6.04 \pm 0.35$ & 2374.7 & $5.38 \pm 0.37$ \\
\hline 2975.8 & $54.97 \pm 2.04$ & 2975.8 & $33.55 \pm 1.42$ & 1786.8 & $7.44 \pm 0.39$ & 2403.5 & $7.14 \pm 0.44$ \\
\hline 2975.7 & $48.40 \pm 1.88$ & 2975.7 & $32.50 \pm 1.40$ & 1852.1 & $8.16 \pm 0.41$ & 2426.4 & $7.68 \pm 0.46$ \\
\hline 3001.7 & $48.80 \pm 1.89$ & 3001.7 & $33.11 \pm 1.41$ & 1878.2 & $7.06 \pm 0.38$ & 2462.7 & $6.50 \pm 0.41$ \\
\hline 3000.7 & $56.20 \pm 2.07$ & 3072.5 & $34.60 \pm 1.44$ & 1947.8 & $8.27 \pm 0.41$ & 2550.2 & $7.47 \pm 0.45$ \\
\hline 3072.5 & $61.70 \pm 2.21$ & 3139.7 & $36.60 \pm 1.49$ & 1969.5 & $9.11 \pm 0.44$ & 2572.0 & $5.93 \pm 0.39$ \\
\hline 3072.5 & $57.20 \pm 2.09$ & 3176.7 & $35.90 \pm 1.47$ & 1986.4 & $8.44 \pm 0.42$ & 2579.8 & $6.92 \pm 0.43$ \\
\hline 3139.7 & $61.00 \pm 2.19$ & 3202.7 & $39.20 \pm 1.54$ & 2003.4 & $8.72 \pm 0.43$ & 2579.7 & $6.50 \pm 0.41$ \\
\hline 3176.7 & $59.10 \pm 2.14$ & 3277.7 & $41.60 \pm 1.60$ & 2067.2 & $9.42 \pm 0.45$ & 2628.0 & $9.00 \pm 0.51$ \\
\hline 3202.7 & $64.20 \pm 2.27$ & 3325.7 & $41.40 \pm 1.59$ & 2092.4 & $9.94 \pm 0.47$ & 2627.7 & $7.80 \pm 0.46$ \\
\hline 3277.7 & $66.20 \pm 2.32$ & 3414.8 & $44.10 \pm 1.66$ & 2142.5 & $8.56 \pm 0.42$ & 2647.7 & $6.10 \pm 0.39$ \\
\hline 3325.7 & $67.10 \pm 2.34$ & 3455.7 & $44.80 \pm 1.68$ & 2195.7 & $9.10 \pm 0.44$ & 2754.8 & $6.26 \pm 0.40$ \\
\hline 3414.8 & $71.80 \pm 2.47$ & 3515.4 & $45.80 \pm 1.70$ & 2261.8 & $10.86 \pm 0.50$ & 2753.7 & $5.40 \pm 0.37$ \\
\hline 3455.7 & $71.80 \pm 2.47$ & 3579.2 & $48.50 \pm 1.77$ & 2299.9 & $11.50 \pm 0.52$ & 2774.5 & $9.22 \pm 0.52$ \\
\hline 3515.4 & $73.30 \pm 2.51$ & 3633.1 & $48.00 \pm 1.75$ & 2299.7 & $11.60 \pm 0.53$ & 2826.7 & $9.80 \pm 0.55$ \\
\hline 3579.2 & $77.50 \pm 2.62$ & 3679.0 & $49.40 \pm 1.79$ & 2374.7 & $10.82 \pm 0.50$ & 2919.2 & $9.44 \pm 0.53$ \\
\hline 3633.1 & $79.10 \pm 2.66$ & 3714.0 & $50.10 \pm 1.81$ & 2403.5 & $12.42 \pm 0.56$ & 2919.3 & $11.30 \pm 0.62$ \\
\hline 3679.0 & $79.60 \pm 2.67$ & 3744.7 & $48.50 \pm 1.77$ & 2462.7 & $12.80 \pm 0.57$ & 2975.8 & $9.11 \pm 0.52$ \\
\hline 3714.0 & $79.30 \pm 2.66$ & 3771.6 & $49.90 \pm 1.80$ & 2511.0 & $11.98 \pm 0.54$ & 2975.7 & $8.30 \pm 0.48$ \\
\hline 3744.7 & $84.40 \pm 2.80$ & 3833.6 & $50.10 \pm 1.81$ & 2572.0 & $13.99 \pm 0.61$ & 3000.7 & $6.60 \pm 0.41$ \\
\hline 3771.6 & $81.90 \pm 2.73$ & 3900.3 & $52.60 \pm 1.87$ & 2579.8 & $13.62 \pm 0.60$ & 3001.7 & $9.23 \pm 0.52$ \\
\hline 3833.6 & $84.20 \pm 2.80$ & 3945.4 & $52.20 \pm 1.86$ & 2579.7 & $13.10 \pm 0.58$ & 3072.5 & $10.40 \pm 0.58$ \\
\hline 3900.3 & $86.00 \pm 2.85$ & 3987.1 & $52.40 \pm 1.86$ & 2628.0 & $13.53 \pm 0.60$ & 3073.6 & $11.40 \pm 0.62$ \\
\hline 3945.4 & $88.10 \pm 2.90$ & 4015.1 & $55.30 \pm 1.94$ & 2627.7 & $14.10 \pm 0.62$ & 3110.6 & $10.20 \pm 0.57$ \\
\hline 3987.1 & $93.80 \pm 3.06$ & 4058.5 & $58.30 \pm 2.01$ & 2647.7 & $12.90 \pm 0.57$ & 3176.7 & $9.80 \pm 0.55$ \\
\hline 4015.1 & $89.30 \pm 2.94$ & 4100.8 & $58.30 \pm 2.01$ & 2754.8 & $14.17 \pm 0.62$ & 3202.7 & $12.60 \pm 0.68$ \\
\hline 4058.5 & $87.70 \pm 2.89$ & 4169.5 & $61.40 \pm 2.10$ & 2753.7 & $13.61 \pm 0.60$ & 3277.7 & $12.10 \pm 0.65$ \\
\hline 4100.8 & $96.70 \pm 3.14$ & 4222.5 & $59.20 \pm 2.04$ & 2774.5 & $14.63 \pm 0.64$ & 3325.7 & $11.70 \pm 0.64$ \\
\hline 4169.5 & $99.60 \pm 3.22$ & 4291.4 & $64.70 \pm 2.18$ & 2773.7 & $14.10 \pm 0.62$ & 3414.8 & $11.50 \pm 0.63$ \\
\hline 4222.5 & $97.50 \pm 3.16$ & 4373.1 & $62.30 \pm 2.12$ & 2826.7 & $14.50 \pm 0.63$ & 3436.7 & $15.70 \pm 0.82$ \\
\hline 4291.4 & $104.50 \pm 3.36$ & 4424.0 & $67.10 \pm 2.25$ & 2919.2 & $16.47 \pm 0.70$ & 3455.7 & $14.20 \pm 0.75$ \\
\hline 4373.1 & $108.30 \pm 3.46$ & 4460.9 & $68.90 \pm 2.30$ & 2919.3 & $16.10 \pm 0.69$ & 3485.5 & $16.10 \pm 0.84$ \\
\hline 4424.0 & $107.40 \pm 3.44$ & 4539.8 & $75.50 \pm 2.48$ & 2975.8 & $15.28 \pm 0.66$ & 3512.4 & $16.50 \pm 0.86$ \\
\hline 4460.9 & $113.20 \pm 3.60$ & 4571.7 & $69.50 \pm 2.31$ & 2975.7 & $16.50 \pm 0.71$ & 3515.4 & $14.60 \pm 0.77$ \\
\hline 4539.8 & $112.60 \pm 3.58$ & 4684.9 & $75.20 \pm 2.47$ & 3000.7 & $15.10 \pm 0.65$ & 3579.2 & $15.50 \pm 0.81$ \\
\hline
\end{tabular}


Table 4. (Continued)

\begin{tabular}{|c|c|c|c|c|c|c|c|}
\hline \multicolumn{2}{|c|}{$1.4 \mathrm{GHz}$} & \multicolumn{2}{|c|}{$2.4 \mathrm{GHz}$} & \multicolumn{2}{|c|}{$4.8 \mathrm{GHz}$} & \multicolumn{2}{|c|}{$8.6 \mathrm{GHz}$} \\
\hline $\begin{array}{l}\text { Day } \\
\text { number }\end{array}$ & $\begin{array}{l}\text { Flux density } \\
(\mathrm{mJy})\end{array}$ & $\begin{array}{c}\text { Day } \\
\text { number }\end{array}$ & $\begin{array}{l}\text { Flux density } \\
\quad(\mathrm{mJy})\end{array}$ & $\begin{array}{c}\text { Day } \\
\text { number }\end{array}$ & $\begin{array}{l}\text { Flux density } \\
(\mathrm{mJy})\end{array}$ & $\begin{array}{c}\text { Day } \\
\text { number }\end{array}$ & $\begin{array}{l}\text { Flux density } \\
\quad(\mathrm{mJy})\end{array}$ \\
\hline 4571.7 & $116.10 \pm 3.68$ & 4728.9 & $74.20 \pm 2.44$ & 3001.7 & $16.84 \pm 0.72$ & 3633.1 & $14.90 \pm 0.79$ \\
\hline 4684.9 & $121.20 \pm 3.83$ & 4768.1 & $77.10 \pm 2.52$ & 3072.5 & $17.80 \pm 0.75$ & 3679.0 & $16.30 \pm 0.85$ \\
\hline 4728.9 & $121.40 \pm 3.83$ & 4799.9 & $77.40 \pm 2.53$ & 3176.7 & $16.90 \pm 0.72$ & 3714.0 & $15.90 \pm 0.83$ \\
\hline 4768.1 & $131.30 \pm 4.12$ & 4837.9 & $83.80 \pm 2.71$ & 3202.7 & $20.20 \pm 0.85$ & 3744.7 & $16.30 \pm 0.85$ \\
\hline 4799.9 & $124.90 \pm 3.93$ & 4850.8 & $77.80 \pm 2.54$ & 3277.7 & $21.60 \pm 0.90$ & 3771.6 & $17.90 \pm 0.93$ \\
\hline 4837.9 & $126.40 \pm 3.98$ & 4870.7 & $77.90 \pm 2.54$ & 3325.7 & $20.70 \pm 0.86$ & 3833.6 & $17.70 \pm 0.92$ \\
\hline 4850.8 & $128.70 \pm 4.04$ & 4936.5 & $81.00 \pm 2.63$ & 3414.8 & $21.30 \pm 0.89$ & 3900.3 & $17.10 \pm 0.89$ \\
\hline 4870.7 & $127.00 \pm 3.99$ & 4997.3 & $83.60 \pm 2.70$ & 3455.7 & $23.10 \pm 0.96$ & 3945.4 & $17.90 \pm 0.93$ \\
\hline 4936.5 & $133.40 \pm 4.18$ & 5024.8 & $83.00 \pm 2.68$ & 3515.4 & $24.80 \pm 1.02$ & 3987.1 & $18.90 \pm 0.98$ \\
\hline 4997.3 & $136.90 \pm 4.28$ & 5050.1 & $86.20 \pm 2.77$ & 3579.2 & $25.40 \pm 1.05$ & 4015.1 & $19.40 \pm 1.00$ \\
\hline 5024.8 & $135.00 \pm 4.22$ & 5093.0 & $86.60 \pm 2.78$ & 3633.1 & $25.10 \pm 1.03$ & 4100.8 & $19.60 \pm 1.01$ \\
\hline 5050.1 & $135.90 \pm 4.25$ & & & 3679.0 & $24.50 \pm 1.01$ & 4222.5 & $19.10 \pm 0.99$ \\
\hline 5093.0 & $143.40 \pm 4.47$ & & & 3714.0 & $24.50 \pm 1.01$ & 4291.4 & $21.00 \pm 1.08$ \\
\hline & & & & 3744.7 & $27.00 \pm 1.11$ & 4373.1 & $21.20 \pm 1.09$ \\
\hline & & & & 3771.6 & $27.10 \pm 1.11$ & 4424.0 & $24.90 \pm 1.27$ \\
\hline & & & & 3833.6 & $26.50 \pm 1.09$ & 4460.9 & $25.50 \pm 1.30$ \\
\hline & & & & 3900.3 & $29.20 \pm 1.19$ & 4539.8 & $25.00 \pm 1.27$ \\
\hline & & & & 3945.4 & $29.80 \pm 1.22$ & 4571.7 & $24.40 \pm 1.25$ \\
\hline & & & & 3987.1 & $31.90 \pm 1.30$ & 4684.9 & $26.70 \pm 1.36$ \\
\hline & & & & 4015.1 & $30.20 \pm 1.23$ & 4728.9 & $28.10 \pm 1.43$ \\
\hline & & & & 4100.8 & $32.00 \pm 1.30$ & 4768.1 & $25.70 \pm 1.31$ \\
\hline & & & & 4222.5 & $32.00 \pm 1.30$ & 4799.9 & $24.80 \pm 1.26$ \\
\hline & & & & 4291.4 & $35.30 \pm 1.43$ & 4837.9 & $31.50 \pm 1.59$ \\
\hline & & & & 4424.0 & $34.10 \pm 1.39$ & 4850.8 & $27.90 \pm 1.42$ \\
\hline & & & & 4460.9 & $38.60 \pm 1.56$ & 4870.7 & $29.90 \pm 1.52$ \\
\hline & & & & 4539.8 & $37.60 \pm 1.52$ & 4936.5 & $30.20 \pm 1.53$ \\
\hline & & & & 4571.7 & $39.60 \pm 1.60$ & 4997.3 & $27.50 \pm 1.40$ \\
\hline & & & & 4684.9 & $40.10 \pm 1.62$ & 5024.8 & $31.90 \pm 1.61$ \\
\hline & & & & 4728.9 & $43.70 \pm 1.77$ & 5050.1 & $31.90 \pm 1.61$ \\
\hline & & & & 4768.1 & $42.70 \pm 1.73$ & 5093.0 & $36.00 \pm 1.82$ \\
\hline & & & & 4799.9 & $39.70 \pm 1.61$ & & \\
\hline & & & & 4837.9 & $43.60 \pm 1.76$ & & \\
\hline & & & & 4850.8 & $45.50 \pm 1.84$ & & \\
\hline & & & & 4870.7 & $47.20 \pm 1.90$ & & \\
\hline & & & & 4936.5 & $48.10 \pm 1.94$ & & \\
\hline & & & & 4997.3 & $43.50 \pm 1.76$ & & \\
\hline & & & & 5024.8 & $47.70 \pm 1.92$ & & \\
\hline & & & & 5050.1 & $46.40 \pm 1.87$ & & \\
\hline & & & & 5093.0 & $49.90 \pm 2.01$ & & \\
\hline
\end{tabular}

